\title{
Nondestructive Evaluations
}

Satish Kulkarni

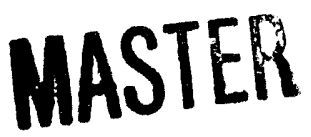




\section{Nondestructive Evaluation}

The Nondestructive Evaluation (NDE) thrust area supports initiatives that advance inspection science and technology. The goal of the NDE thrust area is to provide cutting-edge technologies that have promise of inspection tools three to five years in the future. In selecting projects, the thrust

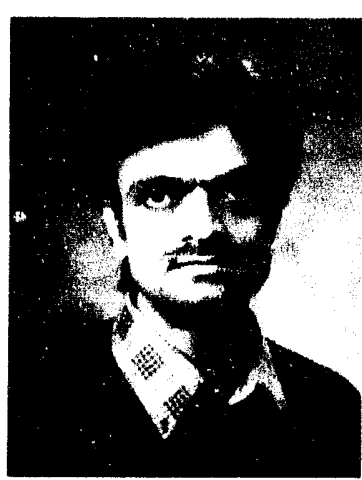
area anticipates the needs of existing and future Lawrence Livermore National Laboratory (LLNL) programs.

NDE provides materials characterization inspections, finished parts, and complex objects to find flaws and fabrication defects and to determine their physical and chemical characteristics. NDE also encompasses process monitoring and control sensors and the monitoring of in-service damage. For concurrent engineering, NDE becomes a frontline technology and strongly impacts issues of certification and of life prediction and extension.

In FY-92, in addition to supporting LLNL programs and the activities of nuclear weapons contractors, NDE has initiated several projects with government agencies and private industries to study aging infrastructures and to advance manufacturing processes. Examples of these projects are (1) the Aging Airplanes Inspection Program for the Federal Aviation Administration; (2) Signal Processing of Acoustic Signatures of Heart Valves for Shiley, Inc.; and (3) Turbine Blade Inspection for the Air Force, jointly with Southwest Research Institute and Garrett.

In FY-92, the primary contributions of the NDE thrust area, described in the reports that follow, were in fieldable chemical sensor systems, computed tomography, and laser generation and detection of ultrasonic energy.

\section{Fieldable Chemical Sensor Systems}

Our objective for this project is to develop diagnostic instruments for quantitative measurements of the levels of chemical contaminants and concentrations in the field or on-line in operating processes. We believe that the integration of Raman spectroscopy and fiber-optic sensors will allow a revolution in chemical analysis by developing the capability for field analysis rather than the more conventional methods requiring extraction of samples for later evaluation in a laboratory environment. We are in the second phase of a two-phase project. In the initial phase, we determined the widespread needs for chemical sensors to measure contaminant levels in liquids and gases and on solid surfaces. We selected Raman spectroscopy as the first system to develop because of its wide applicability as a chemical monitoring technique. During the second phase, we have developed a state-of-the-art micro-Raman spectrometer, designed two unique fiber-optic-based sensors for remote coupling of the spectrometer to either liquid/gas phase samples or solid surfaces, and purchased a new imaging spectrometer and low-light-level detector.

\section{Computed Tomography}

We continue to develop computed tomography $(C T)$ scanners covering a broad range of object sizes. An integral part of this work is to derive the 


\section{Section 8}

reconstruction and display algorithms. The overall goal of this work is to improve the three performance parameters (spatial and contrast resolutions and system speed) that characterize $\mathrm{CT}$ imaging systems. In addition, we have addressed related topics such as elemental or effective- $Z$ imaging, model-based imaging using a priori information, parallel processor architectures for image reconstruction, and scientific visualization of reconstructed data.

In FY-92, we completed the California Competitive Technology Cone-Beam CT Project with Advanced Research and Analysis Corporation as our industrial partner. We continued to work on two other projects: the active/passive CT of radioactive drums and characterization of high explosives for the Pantex plant; and high-purity single-crystal germanium detectors in collaboration with the University of California, San Francisco.

\section{Laser Generation and Detection of Ultrasonic Energy}

Finally, we have developed a facility to generate and detect ultrasonic energy with lasers. Lasergenerated ultrasonics is an attractive alternative to traditional ultrasonic NDE, because it allows remote, noncontacting, ultrasonic NDE. We are developing NDE applications for use on contamination-sensitive components and in hostile environments. Laser ultrasonics has several other advantages, such as broadband excitation, multimode acoustic energy generation, and adaptability to scanning complex shapes.

Satish V. Kulkarni Thrust Area Leader

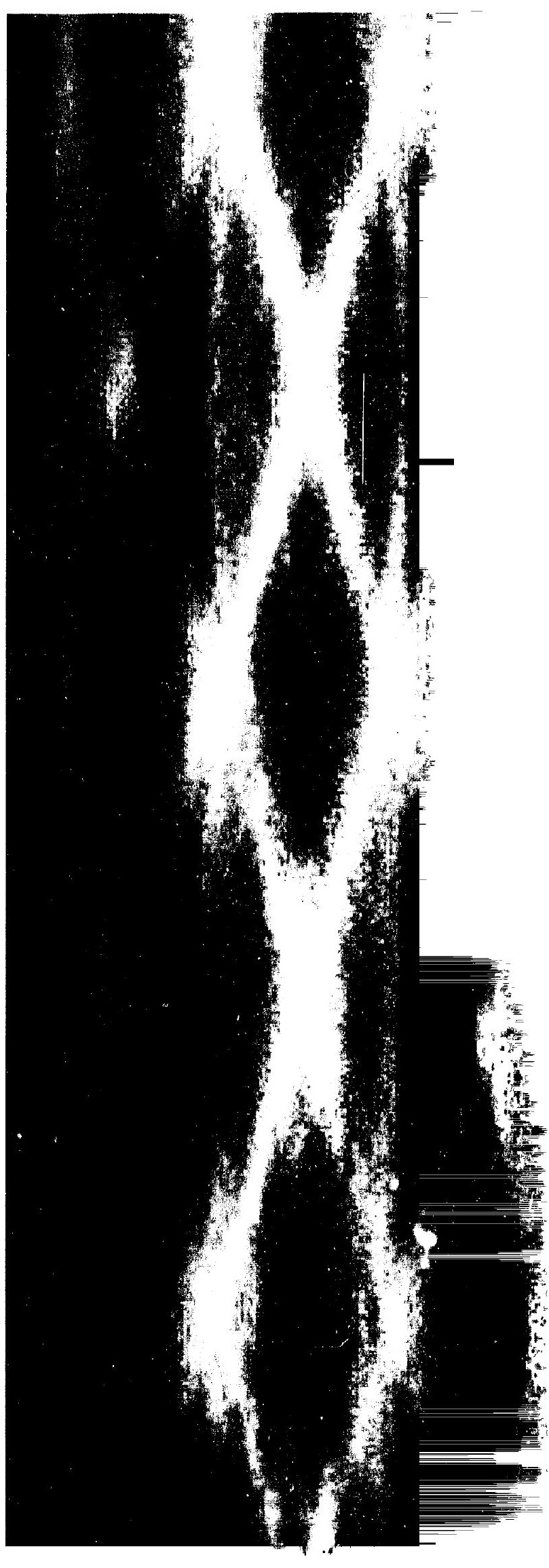




\section{Nondestructive Evaluation}

Overview

Satish V. Kulknmi, Thrust Area Leader

Fieldable Chemical Sensor Systems

Billy J. McKinley and Fred P. Milanovich.

8-1

\section{Computed Tomography}

Harry E. Martz, Stephen G. Azevedo, Daniel J. Schneherk, and

George P. Roberson....

Laser Generation and Detection of Ultrasonic Energy

Grainam H. Thomas 


\section{Fieldable Chemical Sensor Systems}

\author{
Billy J. McKinley \\ Engineering Sciences \\ Mechanical Enģineering
}

\author{
Fred P. Milanovich \\ Environmental Sciences Division \\ Bionedical and Environmental \\ Research Program
}

In the initial phase of this project, we determined the widespread needs for chemical sensors to measure contaminant levels in liquids and gases and on solid surfaces. We selected Raman spectroscopy as the first system to develop because of its wide applicability as a chemical measuring technique.

During FY-91, we developed a state-of-the-art micro-Raman spectrometer capability, designed two unique fiber-optic-based sensors for remote coupling of the spectrometer to either liquid/gas phase samples or solid surfaces, and purchased a new imaging spectrometer and low-light-level detector. During FY-92, we developed two complete systems around these two new sensors and demonstrated the application of the solid surface sensor in the analysis of diamond coatings.

\section{Introduction}

Our objective is to develop diagnostic instruments for quantitative measurements of the levels of chemical contaminants and concentrations in the field or on-line. We believe fiber-optic coupled Raman spectroscopy will make a significant impact in chemical analysis by developing the capability for field analysis, as opposed to the more conventional methods requiring extraction of samples for later evaluation in a laboratory environment. We are in the second phase of a two-phase project.

In the initial phase of this work, we surveyed the needs for sensors, particularly with respect to environmental restoration and waste management concerns. ' The most obvious needs were for chemical sensors that can be used in the field, thus eliminating the costly, time-consuming, and often impossible process of bringing samples to the laboratory for analysis. From our involvement with Nuclear Weapons Complex reconfiguration planning, we also observed a need for on-line or nearline chemical analysis in chemical processing. The combined set of needs ranges from $i n$-situ analysis of contaminants in ground water to on-line monitoring and feedback control at multiple locations along the process line for chemical-processing operations. We are addressing these needs by developing diagnostic instruments and sensor systems that are simple, robust, portable, and sensitiveenough for field operation and decision making.

We have chosen Raman spectroscopy for development for a number of reasons, 1 primarily because of its wide applicability as a chemical sensor. The major problem in the application of Raman spectroscopy is in the signal-to-noise ratio, which is related to the extremely low scattering cross section $\left(10^{-2)} \mathrm{cm}^{2}\right.$ / mol-sr). In a typical measurement, coherent scatter from the laser is $10^{8}$ times greater than the Raman shifted incoherent scatter. The major thrust of our project, therefore, is to create sensors that maximize the Raman scatter and the acceptance angle of the optical system, which collects the scattered light for the spectrometer.

\section{Progeress}

\section{Micro-Raman Spectroscopy Capability}

During FY-91, weestablished a micro-Raman spectroscopy capability. ${ }^{2}$ Although the micro-Raman spectrometer developed represents major progress in our facilities and capability, it is limited in various ways: (1) it can only accept 0.5 -cm-dia samples; (2) it re quires considerable alignment of the optical compernents on a regular basis; and (3) the old spectrometer has considerable scattering noise, and the detector system's sensitivity and noise figure are not as goxnd as that of more modern detectors. 


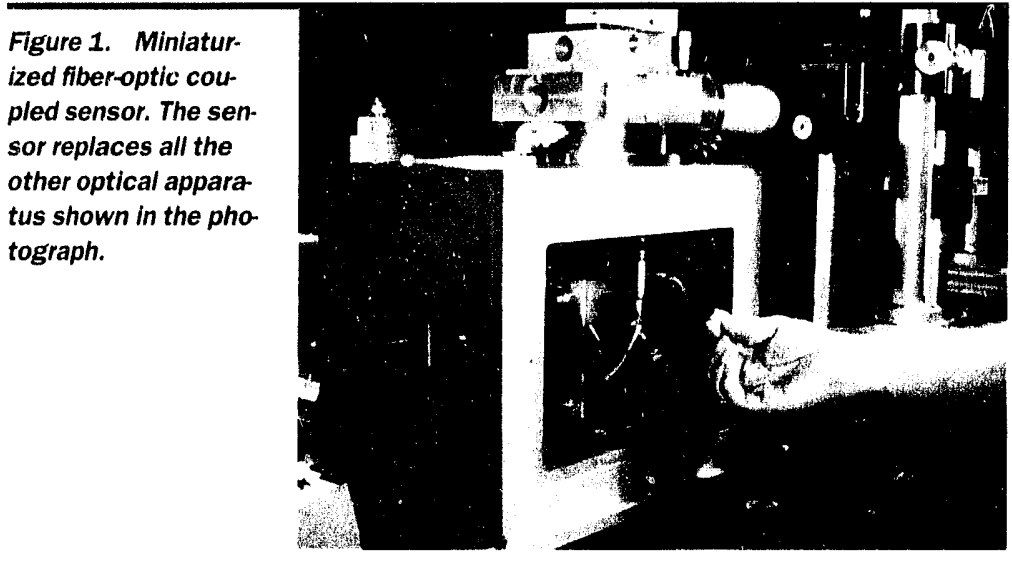

Figure 2. Illustration of the principle of operation of the one-sided Raman sensor for solid sur. faces.

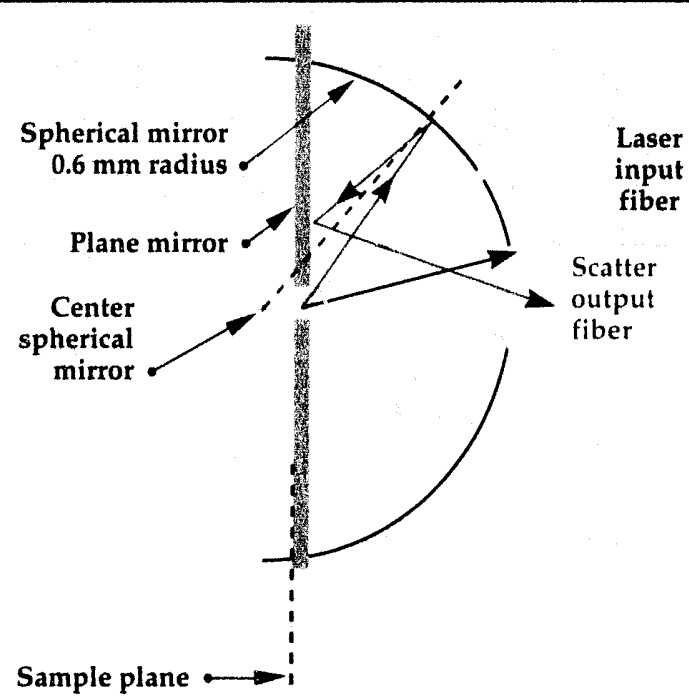

Figure 3. Comparison of data for onesided (NIG) sensor and micro-Raman system.

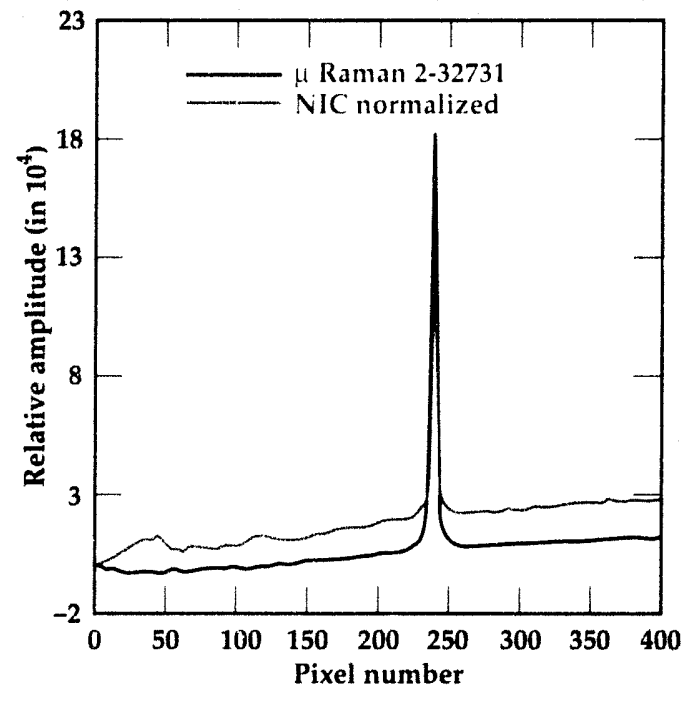

To correct the deficiencies of this system and to modify it to serve as the testbed for more advanced systems, we purchased an imaging spectrometer and a lesi-light-level, liquid-nitrogencooled, two-dimensional charge-couple-derice detector. The combination of these new instruments is a significant advancement in our capability to address multipoint, high-spectral-resolution, broad-bandwidth spectral analysis and the very demanding low-light-level recording issues assorciated with Raman spectrosicopy.

\section{Fiber-Optic Remote Coupling Devices}

As previously stated, the core objective of this research is to develop the instrumentation necessary to perform field analysis. This includes two fiber-optic-based devices for remote coupling of the spectrometer to the sample. The first, to be used in analysis of solid surfaces, is referred to as the one-sided sensor; the second, for analysis of trace concentrations in solutions, is a liquid-core optical waveguide. The liquid core serves as the sample cell and as a low-loss light transmission line. The waveguide not only retains the laser excitation light, but is an efficient concentrator of the Raman scatter. Both of these designs are discussed further below.

One-Sided Sensor. Our goals with the onesided sensor are to achieve a major advance in miniaturization of instrumentation; to eliminate numerous interactive, optical-alignment adjustments; to accommodate large solid-surface evaluation; and to allow more practical, robust, quantitative field applications of spectroscopy. The fiber-optic-coupled sensor shown in Fig. 1 replaces all the other optical apparatus shown in the photograph, reducing the system size by at least a factor of 100. The fiber-optic sensor needs no adjustments; only its photons move.

Figure 2 shows the principles of the sensor operation: light from the laser is focused onto the surface of the object of interest through an aperture $(20-\mu \mathrm{m}-\mathrm{dia})$ in a plane mirror. The mirror is a thin $(2-\mu \mathrm{m})$ aluminum-coated polymer membrane. The plane mirror is off-set from the center of the spherical mirror at the appropriate distance for maximum light-gathering efficiency. Light scattered back through the aperture is collected directly by the fiber up to an incident angle roughly equal to the numerical aperture (N.A.) of the fiber (the acceptance angle of the fiber is proportional to the N.A.). Scattered light incident at greater angles is reflected by the two mirrors back to the fiber at a 'shallower' angle within the N.A. of the fiber.

The prototype for this sensor has been evaluated, and a comparison is made with the microRaman system (Fig. 3). Although preliminary results are very good, further improvements are possible by appropriate optical filtering to elimi- 
nate the Raman scatter being produced in the coupling tibers.

Liquid-Core Optical Waveguide Design. Our goal with the licpuid-core optical waveguide is to make a major breakthrough in lowering the threshold achievable in on-line analysis for trace concentrations in aqueous solutions.

As mentioned earlier, the major limitation of using Raman spectroscopy for chemical analysis is the very low molecular Raman scattering cross section. For analysis of low (ppm) concentrations in solutions, the problem is orders of magnitude more severe than for concentrated solutions.

Toovercome this limitation, we have designed the optical system to maximize the sampling interaction path length in the solution and the acceptance angle of the scattered light returned to the detector. The test bed for evaluating these new waveguides is shown in Fig. 4.

\section{Future Work}

Our plans for future work include three areas:

(1) Optimization of optical system throughput. Greater efficiency can be achieved by optimizing the transfer of light from the sensors to the spectrometer. The spectrometer has a numerical aperture of 24 and a minimum slit width of $10 \mu \mathrm{m}$, which set the boundary conditions for the entire optical system. We have begun the optimization design for the one-sided sensor. Many of the limitations and the optimization methods apply directly to the liquid-core waveguide as well.

(2) Testing the liquid-core optical waveguide (coll). We intend to test the system on two categories of problems: (1) analysis of trace contaminant concentrations in groundwater, and (2) chemical process monitoring and feedback control. In the latter case, we will choose a chemical from the uranium-processing line identified in the Nuclear Weapons Complex Recontiguration Study, as the model for the analytical system in the de-

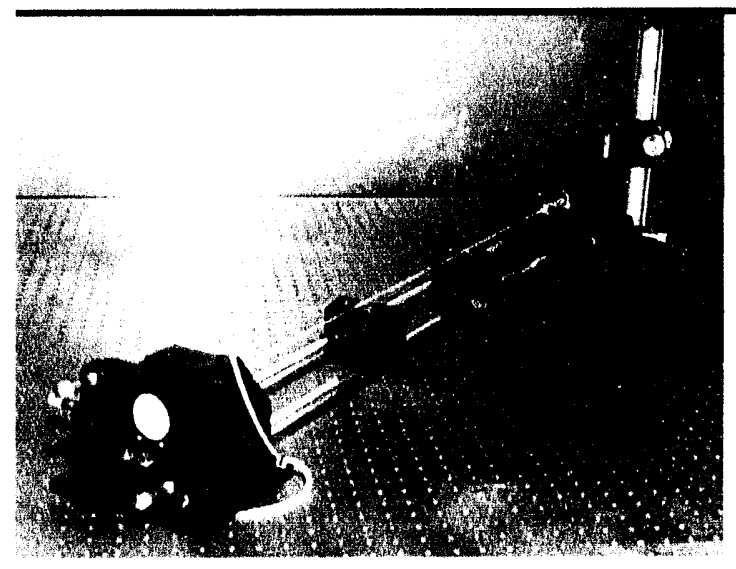

Figure 4. Testbed for evaluating new waveguides. sign of the cell. We will also develop the spertral analysis algerithms that will be necessary in a feedback control loop.

(3) Spectroscopy. To complete this phase of the project, creating a capability for Raman analysis, we must develop more understanding and have more experience in spectroscopic analysis. The first steps have been taken in the purchase of new spectral-analysis software and a Raman specira-comparative database: We are working with specific examples, such as the diamond coating evaluation.

\section{Acknowledgements}

The authors would like to acknowledge John Lutz and Sang Sheem for their contributions to this project.

1. B.f. McKinley, I:I. Milanowid, M.S. Angel, and H.K. McCue, "Fieldable Sensor Systems for Environmental Contaminants," Fingincering Resedoldamet

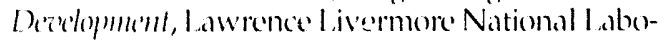
ratory, Livermore, California, UCRL-53868-9)(1,7-9 (|c) (9).

2. B.J. McKinley and FIP. Milanovich, "Iïcldable Chemical Sensor Systems," Enginering Resemell, Derelopment, and Tichmolegyly, Lawrence livermore National Laboratory, I ivermore, California, UCRL$53868-91,7-1(1992)$. 


\section{Computed Tomography}

Harry E. Martz

Engine ring Sciences

Mechlunical Engineering

Stephen G. Azevedo

Engineering Resenrch Dizision

Electronics Engineering
Daniel J. Schneberk

Applications Systems Division

Computation Directorate
George P. Roberson

Defense' Sciences Engine ring Division

Electronics Engineering

We are developing several data-acquisition scanners for computed tomography (CT), along with associated computational techniques for image reconstruction, analysis, and display. This report describes recent progress in active and passive $C T$, cone-beam $C T$, high-energy $C T$, and specialized applications research. We have sought to advance the state of the art in CT technology, while at the same time actively supporting programs at Lawrence Livermore National Laboratory and new business initiatives. Our goal is to provide reliable and efficient nondestructive evaluation techniques for use in probing the internal structure of fabricated objects and materials associated with a broad spectrum of applications.

\section{Introduction}

Nondestructive evaluation (NDE) is being used in an ever broadening array of industrial and military applications. One area in rerent years where growth is evident is computed tomography (CT). First used in the 1970's as a medical diagnostic tod, CT was adapted to industrial and other nonmedical purposes in the mid-198()'s. Standard radiographic techniques, such as single projection radiography, hide crucial information: the overlapping of features obscures parts of these features, and the depth of the features is unknown. CT was developed to retrieve three-dimensional (3-D) information.

For CT, several radiographic images of the object are acquired at different angles, and the intensity information collected by one or many detectors is processed in a computer. The final 3-D image, generated by mathematically combining these images, gives the exact locations and dimensions of internal features within the object, as well as external details. Over the past six years, we have worked on research and development (R\&D) of many CT topics, concentrating on three main areas: (1) scanners, (2) software tools, and (3) applications. " 4
Two years ago, we began R\&D on a combined active and passive computed tomography (A\&PCT) system. 1,2

In this report, we describe our major progress in A\&PCT, cone-beam CT and high-energy CT. We also present advances in the application of these and other capabilities for both Lawrence Livermore National Laboratory (LLNL) programs and business. Lastly, we outline our future plans.

\section{Progress}

\section{A\&PCT Research}

Characterization of mixed (radioactive and hazardous) wastes requires that the identity and strengths of intrinsic radioactive sources be determined accurately. In collaboration with LLNL's Nuclear Chemistry Division, we havedeveloped a three-phased plan to address the nondestructive assay (NDA) of 2)8-L (i.e., 55-gallon) drums. These phases are (1) experimental A\&PCT research and development, (2) simulated A\&I'CT research and development, and (3) determination of minimumdetectable limits vs waste-matrix attenuation. We report here on the experimental and simulated 
A\&I'T efforts. The determination of minimumdetectable limits es waste-matrix attenuation offort was fincted by the Office of Safeguards and Security and is described elsewhere."

A\&PCT Scanner. Experimental data were acquired on a small-scole canister containing mock wastes and two passive sources, 95- 4 C i $133 \mathrm{Ba}$ and

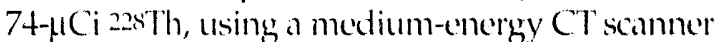
(MECAT) built at LLN NL. 11.12 These data were used to investigate (1) ACT, to obtain images that represent cross-sectional attenuation maps of a wastecanister's contents; (2) IC'T, to lociate and determine

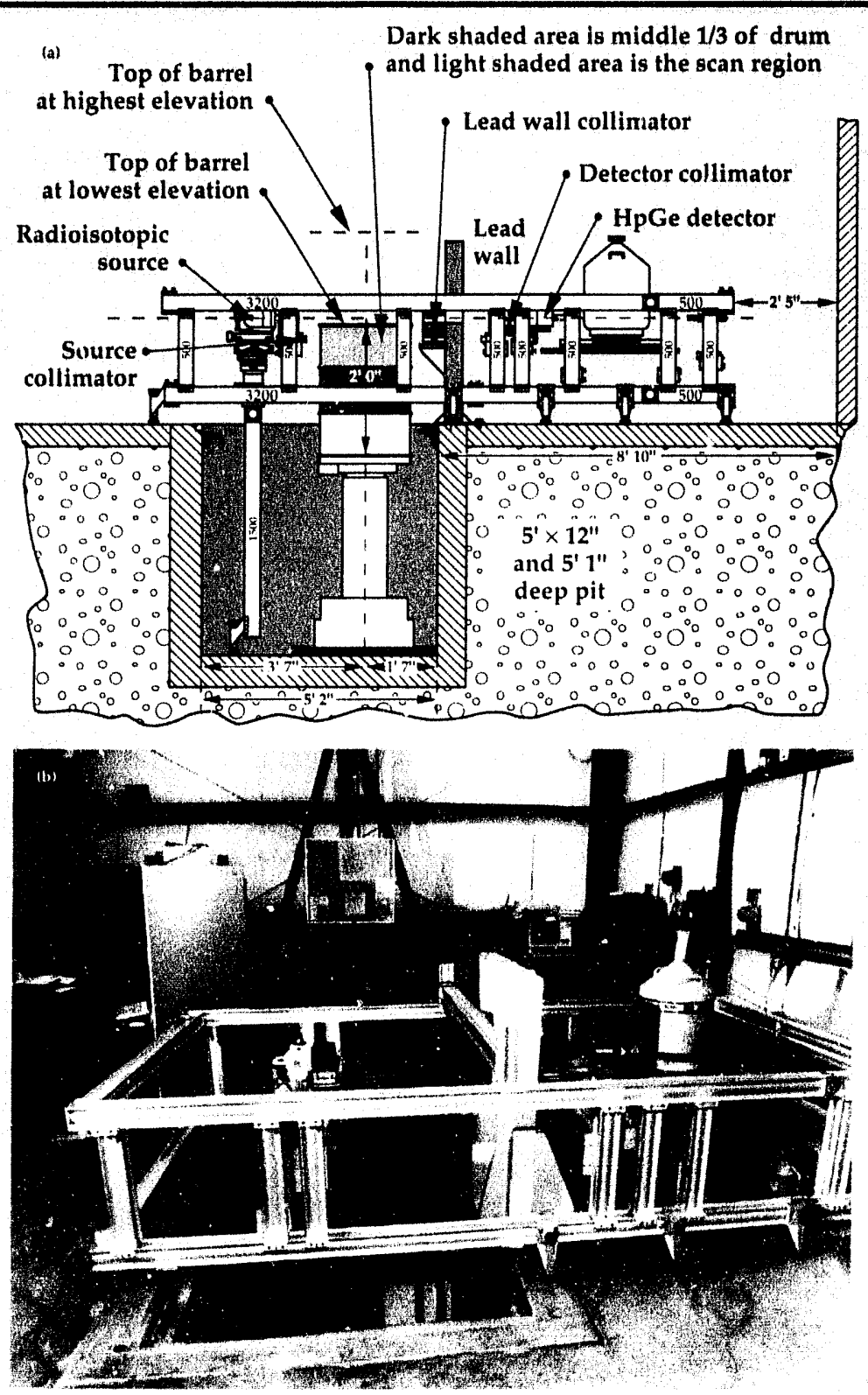

Figure 1. (a) ACT and PCT prototype scanner design; (b) scanner photo, showing construction to date. the identity of any rodioisotepic sources present; and (3) ACT data, to correct the PC'T data so that accurate source strengths can be determined.

Our experimental results reveal that $\Lambda C \mathrm{C}$ scans properly map the canister's attenuating matrix and, when coupled with I'CT scans, yield yuantitative source strengths. 13 Preliminary results suggest that heavy-metal content, which is larger than the volume-element size imaged, may be identified. These encouraging results have led us to design and construct a full-scale, 2(18-L prototype A\&I'CT drum scanner.

The full-scale, 2()8-L prototype A\&PCT drum scanner uses a single, high-purity germanium (HIPC) detector of the type used in nuclear spectroscopy measurements. This scanner's construction is scheduled for completion early in 1993. The scanner design and progress to date are shown in Fig. 1. This scanner will be used to better explore and understand the relationships among the four most important CT performance or resolution parameters-spatial, contrast, energy, and temporal (or speed)-from the point of view of assaying nuclear waste drums for radioactive content. The definition of contrast resolution differs for the A\&PCT measurements. In the former, it is a measure of attenuation differences that can be observed; in the latter, it is a measure of radionctive strength differences. Speed includes data-acquisition and analysis time.

Limits to improving the I'CT activity results include geometrical uncertainties caused by the collimator's angular cone of acceptance, photon scattering, lack of sufficient counts, the randomness inherent in photon counting, poor detection efficiency, the energy resolution required, system noise, data-acquisition, and times required for data analysis. Quantitative assays using PCT are further complicated by the need for attenuation corrections, which are obtained from the ACT data. Unfortunately, these data are limited by many of the same performance parameters.

Reconstruction Technologies. We have developed A\&PCT image-reconstruction and simulation algorithms to better characterize mixed-waste drums, in collaboration with Laboratoire d'Electronique de Technologiect d'Instrumentation (LETI) in Crenoble, France, and the University of California at San Francisco (UCSF). The A\&PCT image reconstruction and analysis process consists of mapping the activity of intrinsic radioactive sources, using P'CT data, and correcting this 
data for attenuation, by using an attenuation matrix obtained from an $A C T$ scan. Simulated data are necessary to better understand A\&I'C T' reconstruction algorithms and measure their performance, and to better interpret experimental data. The simulation program is based on a forwardprojection algorithm ${ }^{14}$ that discretely computes the projections; i.e., integrated counts per unit time per unit volume, of an emitting object attenuated by a user-specified matrix. We use two algebraic, iterative, A\&PCT reconstruction codes: a v : h.hted-least-squares, stecpest-descent (WLS-iD) algorithm and a maximum-likelihoot expectationmaximization (MLEM) algorithm. 14

We studied three simulated phantoms: (1) a large homogeneous source included in a large homogeneous attenuator; (2) a mock-waste drum involving small sources; and (3) a spatial-resolution phantom. ${ }^{15}$ All three examples involve attenuation but are not strong enough to cause missing data, and the last example includes noise. The noise is generated to match experimental uncertainties. Results showed that both reconstruction algorithms recover the activity values to within experimental uncertainty (counting statistics). The WLS-SD algorithm produces more spreading of the activity over multiple pixels, but also performs slightly better than MLEM (i.e., it gives more accurate activity values) in the case of noise. We are working on a number of improvements in these algorithms; e.g., incorporation of collimator geometry, addition of the effects of very strong attenuation, and optimization of the code for speed and activity accuracy. We are also investigating other algorithms.

A\&PCT Applications. In addition to continuing experiments on the small-scale canister of mock waste, we studied the attenuation of both passive sources by a uniformly attenuating $C u$ cylinder. A representative ACT image of the Cu cylinder with both passive sources is shown in Fig. 2 . Note that the locations of the cylinder and both passive sources are easily visible. It is also interesting to point out the $30 \%$ difference in the $\mathrm{Cu}$ cylinder wall attenuation value. We found that this difference may be due to a wall thickness variation of $\sim 1 \mathrm{~mm}$ from one side of the cylinder to the other, and porosity. A portion of the resultant change in the wall attenuation value can be attributed to partial volume effects (due to the crude 3-mm spatial sampling) instead of a noticeable change in wall thickness.
For the attenuated PCT scan, a combination of three factors yielded noisy net passive projection data: (1) $3-\times-3-\mathrm{mm}$ aperture, (2) the short datoacquisition time (150s), and (3) the attenuation of the passive sources by the copper cylinder $(\sim 10.1 \mathrm{~cm}$ o.d. and $\sim 8.9 \mathrm{~cm}$ i.d.). The passive source's' energy peaks were within the Compton and background distributions. It is important to point out that the method of extracting the net-passive projection data (gross counts minus spectral background) from the gross projection data is not adequate for low-count-rate PCT data, and resulted in meaningless net-peak intensities for the passive projection data sets. Figure 3 shows a representative comparison between the gross and net projection data for the 228Th source at $238 / 240 \mathrm{keV}$, and for the ${ }^{133 \mathrm{Ba}}$ source at $384 \mathrm{keV}$. Neither the $22 \mathrm{TTh}$ nor the $133 \mathrm{Ba}$ net projection data reveal any

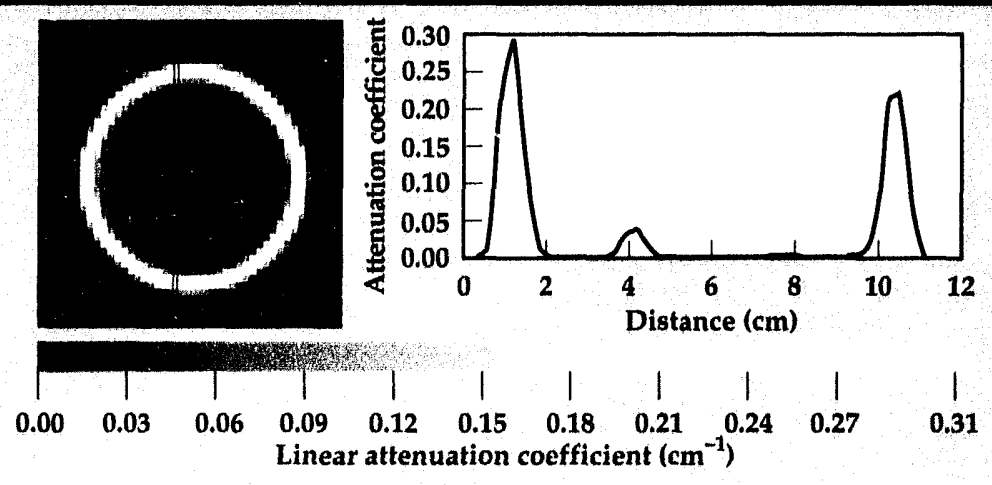

Figure 2. Representative ACT image of a Cu cylinder with passive sources. A 1-D profile of this data is on the right.

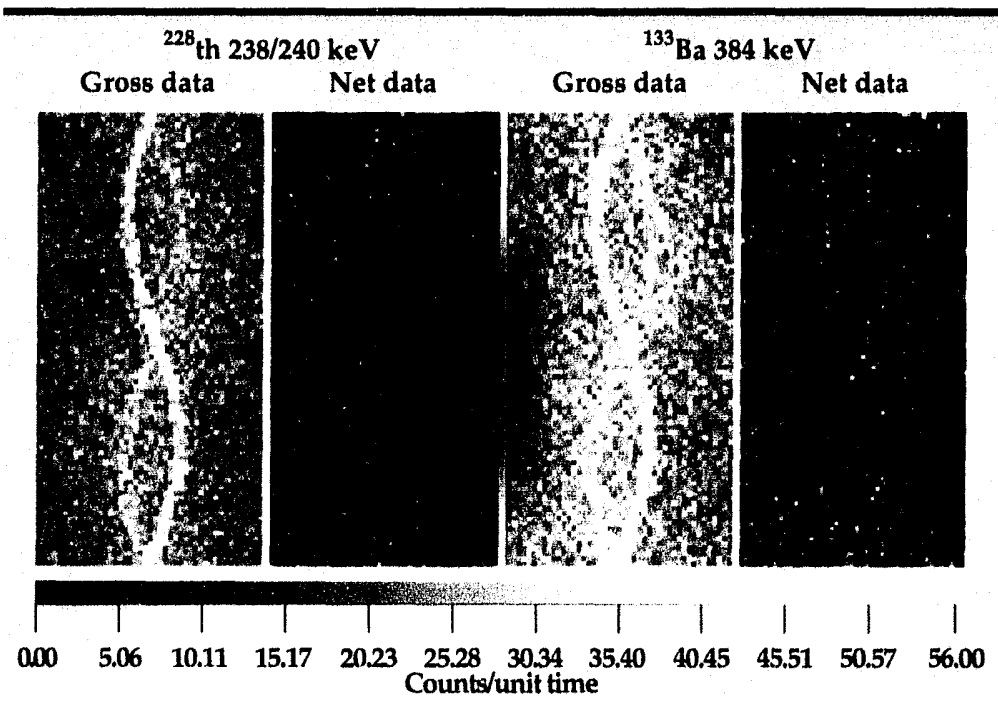

Figure 3. Representative gross and net passive sinogram data at $238 / 240 \mathrm{keV}$ for the ${ }^{228} \mathrm{Th}$ source, and at $384 \mathrm{keV}$ for the ${ }^{133} \mathrm{Ba}$ passive source. 
intermal source distribution mor did the final attenwation corrected PCT images (see Fig. 4). The resultant, noiss, passiese sinogram data are due to a low count rite

Since the net projection data did not result in passive source identification or localization of activity, we analyed the gross projection data. As expected, these data are distorted. For example, the gross 133 Ba projection A\&I'CT WIS-SD-reconstructed PCT-image data (Fig. 4) appear to have three internal source distributions: (1) a 1.33 Ba passive source at the lecation, as expected from the ACT image in Fig. 2; (2) an apparent 1.33 Ba source at the lecation of the 22xTh source, and (3) an apparent 133 Ba ring source. Only the first distribution is real; the latter two are artifacts and are very misleading when these data are analyzed for the 133Ba source activity. For comparison, the results of PCT scan data without attenuation (i.e., no Cu

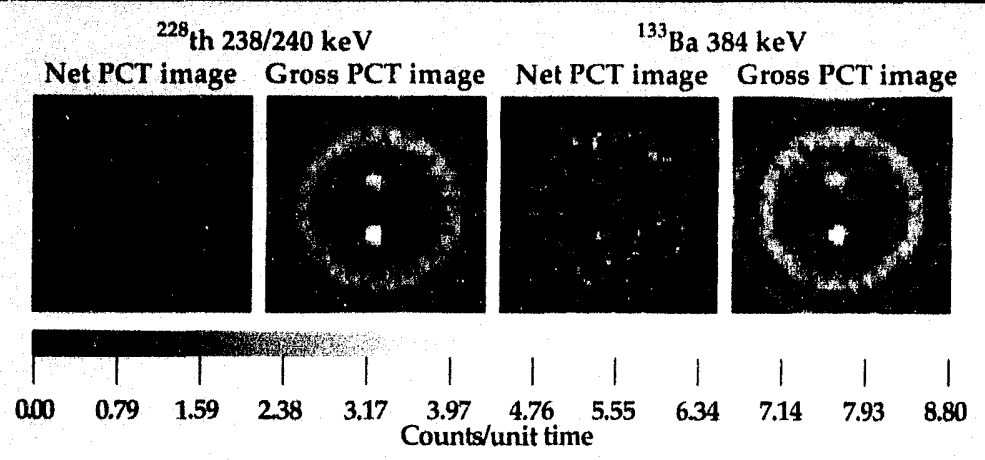

Figure 4. Corrected PCT images obtained by reconstructing the net and gross sinogram data, using the WLS/SD algorithm.
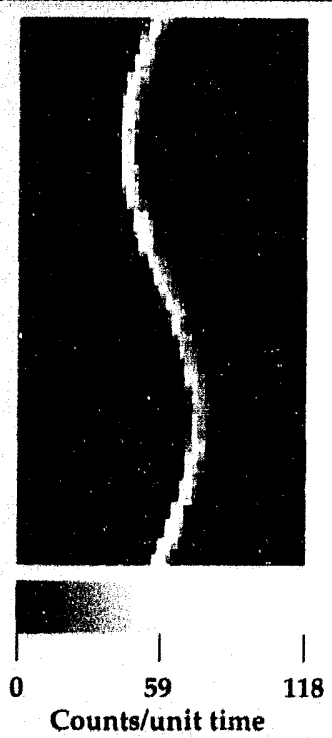
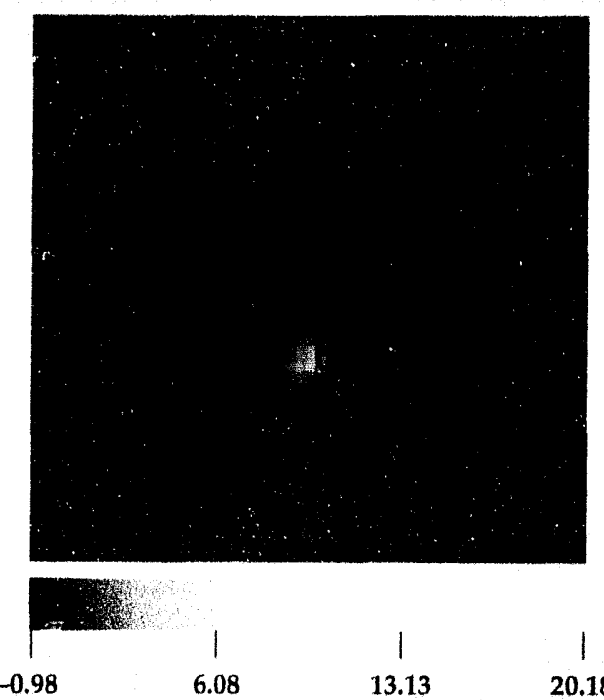

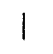
$\begin{array}{cc}6.08 & 13.13 \\ \text { unts/unit time/unit Voxe }\end{array}$

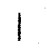

20.18
Figure 5. Representative unattenuated passive sinogram and PCT image data for the ${ }^{133} \mathrm{Ba}$ source at $384 \mathrm{keV}$. cylinder or 2xTh passive source) are shown in Fig. 5. We are investigating the use of other gamma-ray spectrum processing methods that will improve the extraction of the net-peak data from noisy gamma-ray spectra. Using a better method is important, since we expect low-count-rate data to be the norm in mixed-waste drum assay scans containing LL.W amounts of activity.

\section{Cone-Beam CT Research}

We are expanding our research in cone-beam CT imaging methods. We begin this section with an introduction to cone-beam CT imaging, and follow with our progress during FY-92.

Computed tomography of the 1970's and early 1980 's has been an inherently two-dimensional (2-D) process. Typically, a single detector or linear [onc-dimensional (1-D)] array of detectors is used to gather $x$ ray-attenuation transmission gauge measurements through the object under inspection. One gauge measurement is called a ray sum; multiple ray sum measurements along a single line are called a projection. Many projections are acquired at various angles about the object, but always through the same cross-sectional plane (see Fig. 6a), creating a 2-D data set called a sinogram. Image reconstruction of such 2-D sinograns usually involves filtering and backprojection operations that are well characterized and understood, and results in 2-D, cross-sectional images.

With the ready access of microfocus (spot sizes of about 1 to $50 \mu \mathrm{m}$ ) $x$ ray machines, good 2-D (planar) $x$ ray detectors and improved video technology in the last decade, CT research has concentrated on direct 2-D projection (or radiographic) measurements and one-step, 3-D, volumetric image-reconstruction methods. This speeds up the data-acquisition process (since multiple slices are acquired simultaneously) and results in a more efficient use of the $x$ ray source. Until recently, $2-D$ projection measurements were acquired with the $x$ ray source far from the detector, so the radiation penetrates the object with parallel-beam rays and standard2-D image-reconstruction methods could be used (Fig. 6b). Currently, the more interesting case is to acquire projection data with the source close to the object and detector. This mode results in cone-beam $x$ ray imaging (Fig. 6c). Cone-beam CT allows the use of geometric magnification to improve spatial resolution, and it makes the most efficient use of the source radiation. Problems to be solved with cone-beam CT include scanner alignment and the need for more complex image-re- 
construction algorithms. We have addressed these problems and will show some results throughout the following sections.

Cone-Beam CT Scanners. Several of our CT scanners are inherently cone-beam systems. 2.5 Our recent collaborative work on cone-beam imagereconstruction methods (discussed below) has enabled us to use these scanners in a variety of new applications, and to take full advantage of improvements in source and detector technology. This has resulted in a better understanding of the components involved in cone-beam scanners.

A crucial component of many cone-beam scanners is the scintillator, the mechanism for converting the $x$ ray photons into visible light. Recent developments in glass scintillator fabrication and manufacture provide the potential for increased performance of our lens-coupled, camera-based scanners such as the MicroCAT and the highenergy CT scanner (HECAT). To examine the possibility of increased scanner performance, we have applied our lens-coupled scanner's to evaluate this new type of scintillator glass, called Lockheed high density (LHD) glass. In general, we seek to establish the spatial resolution, speed, energy response, and contrast limits of this glass for the wide variety of different digital radiography and CT applications we usually encounter within the Nondestructive Evaluation section.

The promise of this new glass is high trightness and high spatial resolution, a combinatior which is not readily available without substantial cosis. High brightness can be obtained with off-the-shelf image intensifiers, or image-intensified charged couple de' vices $\left(C C D^{\prime} s\right)$. Often these imagers include spatial distortions due to their physical shape, and image intensification components. Distortions must be accounted for before meaningful CT images can be obtained.2 Furthermore, intensifier-based CГ scanners can have other limitations due to the actual spatial resolutions that can beobtained.'

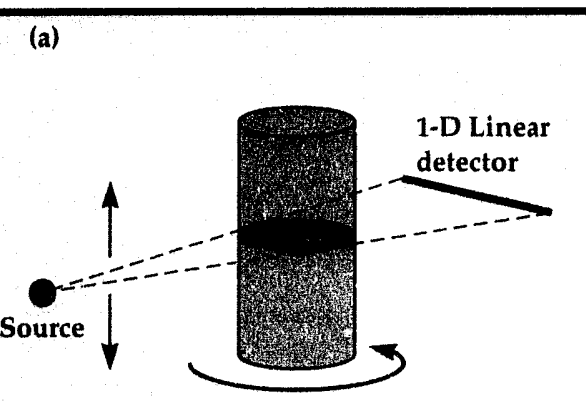

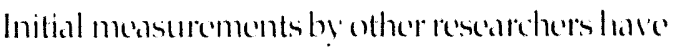
shown that the 1.11D) ghass composices a flat-fiedd, distortion-free projection image with at least 25to $35-\mu m$ (or $2(1)-$ to $14-1 \mathrm{p} / \mathrm{mm}$ ) inherent spotial resolution, and is bright enough for standard, nomintensified, visible-light C(D) comeras." We are currently evaluating a (ohu 4910 comera, operated in two modes, variable integration mode and RS-17) mode. We have also used the ER210 lmage-Intensified Silicon-Intensified Target (SIT) camera and a l'hotometrics $(\mathrm{H} 20) \mathrm{CCD}$-based camera to further explore the propertics of the LHI) glass at high energies ( 4 and $9 \mathrm{MoV}$ ). If the reported properties can be realized routinely on general purpose, lens-coupled, $x$-ray imaging systems, with a variety of cameras, then a relatively inexpensive and high-performance, $x$-ray imaging alternative has been established.

To evaluate the new scintillator glass over a broad range of energies, we ran three separate series of experiments at the following energies: (1) at 90 to $130 \mathrm{kVp}$, using the MicroCAT scanner; (2) at 206 ; to $320 \mathrm{kVp}$, using a IPHILLIPS 32()-kVp medium energy source; and (3) at 4 and $9 \mathrm{MeV}$ using two VARIAN Linatrons and the HECAT scanner detector. In all three experiments, we used the scanners in a similar fashion to that with their original scintillating glass materials. The only difference was that the original scintillators were replaced with the new, clear, LHD glass scintillators.

Our low-energy studies have produced data with spatial resolutions $(\sim 1+$ to $20 \mathrm{lp} / \mathrm{mm})$ consistent with earlier results, ${ }^{17}$ but with a slight variation in technique. Scintillating glass can be fabricated as a clear sheet, or drawn into fiberoptic bundles. We are interested in detectors that can support cone-beam imaging modes, and consequently restricted our evaluations to the clear, LHD scintillator material. It is known that conebeams of $x$ rays impinging on a fiber-optic scintillator will induce cross-talk in the fibers, and result (b)

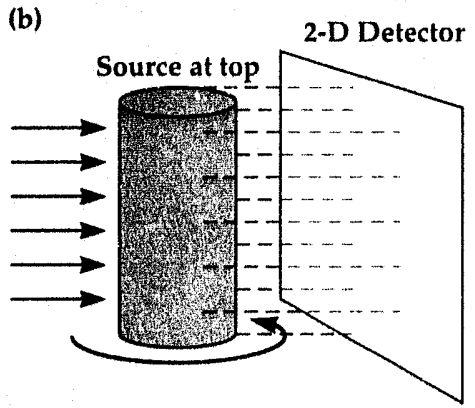

(c)

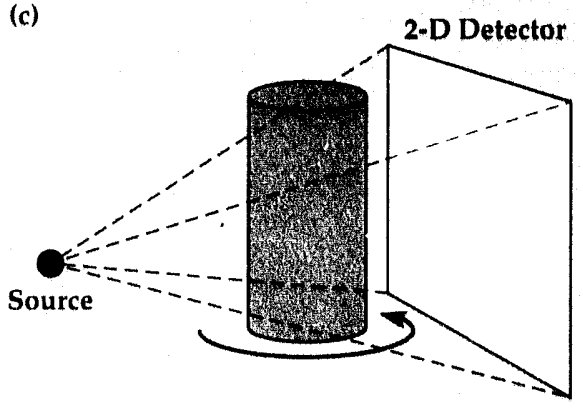

Figure 6. Methods of acquiring 3-D Cr data: (a) multi-slice $C r$ by acquiring each 2-D cross-sectional slice independently; (b) 3-D parallel Cr with a 2-D detector and source at infinity (one rotation and standard reconstruction codes are used); (c) cone-beam $C r$ by moving the source close to the object. New reconstruction algorithms are needed to process this data. 
Figure 7. Digital ra diograph of hollow thermocouple plug, taken with Micro. CAT system.
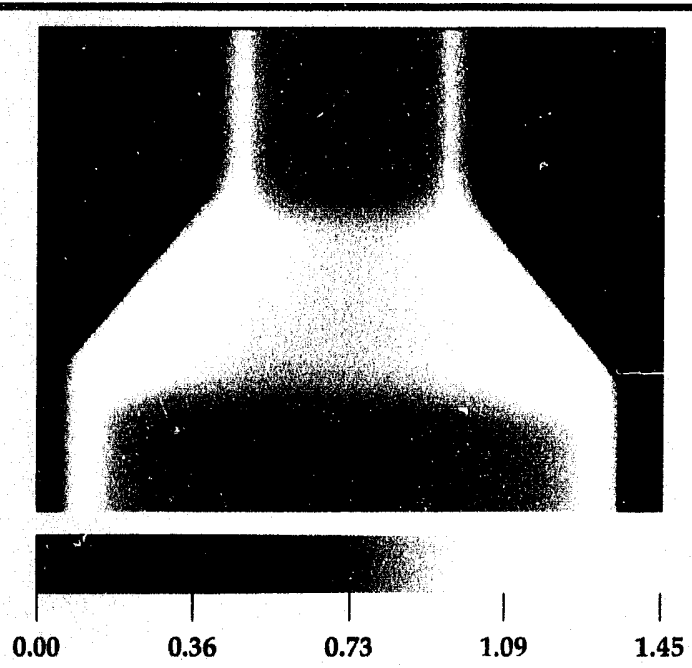

Figure 8. The spa tial resolution performance of MicroCAT studied by analysis of a linopair gauge.
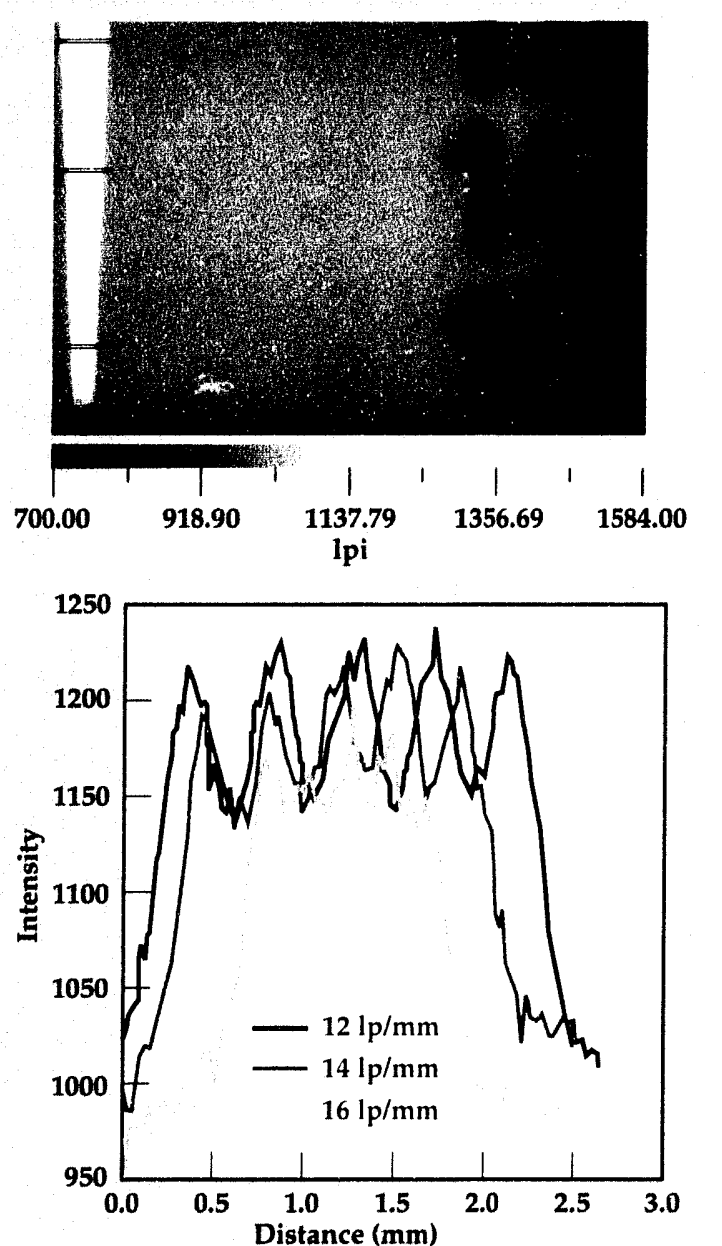

in a loss of spatial resolution as cone angle increases. In In addition, the unprocessed image can be viewed directly, without any subtraction of the cross-hatch in the fiber-bundle usually present in unprocessed images from fiber-optic scintillators. Figure 7 is a digital radiograph of a hollow thermocouple plug cover, taken on our MicroCAT system. One surprising result of this investigation is the increased spatial performance obtained when the focal plane of the camera is positioned inside the scintillator glass, as opposed to the back face plane of the glass. The advantages of this technique are visible in the image in Fig. 7. However, this added clarity is at the cost of some brightness, since some of the scintillator material is not in focus for the camera.

The MicroCAT scanner's spatial resolution performance using the LHD glass scintillator was further studied by analysis of a line-pair gauge (Fig. 8). There was almost 110 magnification involved in this exposure $(M=1.1)$, and with our Micro-Focal system (10- $\mu \mathrm{m}$ spot sizes), blur due to finite spot size is effectively eliminated from the system. ${ }^{14}$ As illustrated in this figure, the loss in spatial modulation is less than $50 \%$ from 12 to $14 \mathrm{lp} / \mathrm{mm}$, and there is significant modulation at $16 \mathrm{lp} / \mathrm{mm}$. With the micro-focal source, magnifications of 2 to 3 can be achieved with a minimum of source blur, which can easily extend the spatial resolution of this scanner into the 18 - to 2()$-\mathrm{lp} / \mathrm{mm}$ range. We have scanned different objects, and our results have shown increased performance compared to scans with the fiber-optic scintillator used previously. A comparison of the enhancement in the new LHD glass scintillator and other differences with the older fiber-optic scintillating glass for a pinch-weld CT study are shown in Fig. 9.

The spatial resolution performance reported by others ${ }^{\text {? }}$ has been substantiated by some of our preliminary studies using the LHD glass scintillator at medium and high energies as well. For example, a representative digital radiograph of a $\sim 3-\mathrm{cm}$ turbine blade acquired with a lens-coupled, Cohu 4910, CCD camera detector system and a 27()-kVp $x$ ray spectrum from a Philips mediumenergy machine source (spot size $\sim 0.4 \mathrm{~mm}$ ) is shown in Fig. 10. We estimate the spatial resolution of this digital radiograph to be on the order of $7 \mathrm{to} 10 \mathrm{lp} / \mathrm{mm}$. 


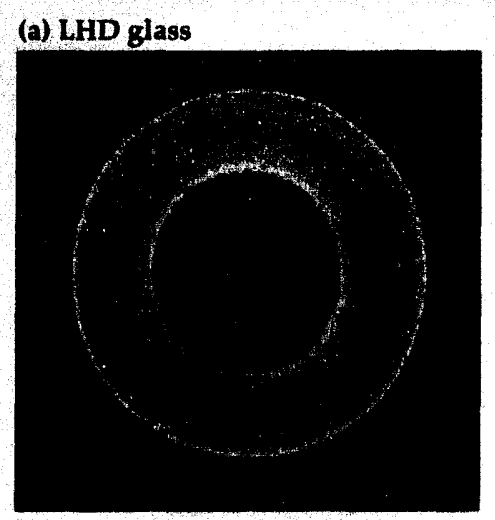

(b) 2-year-old SDD glass
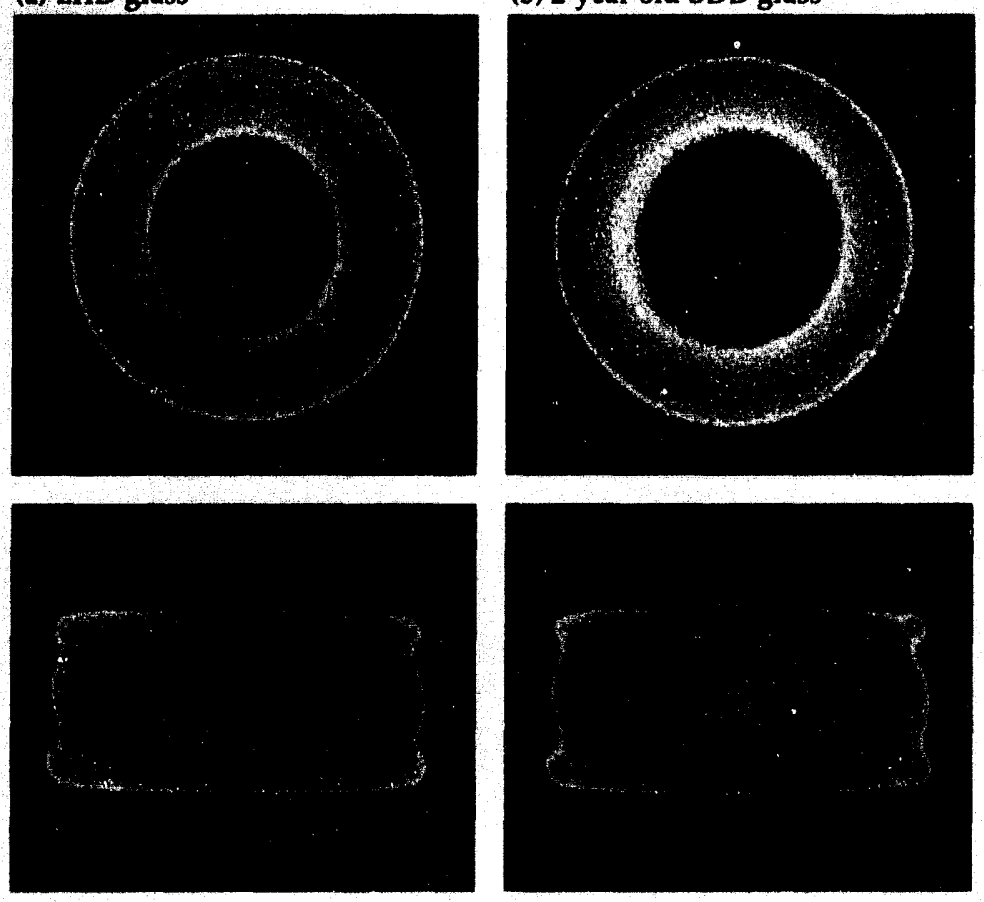
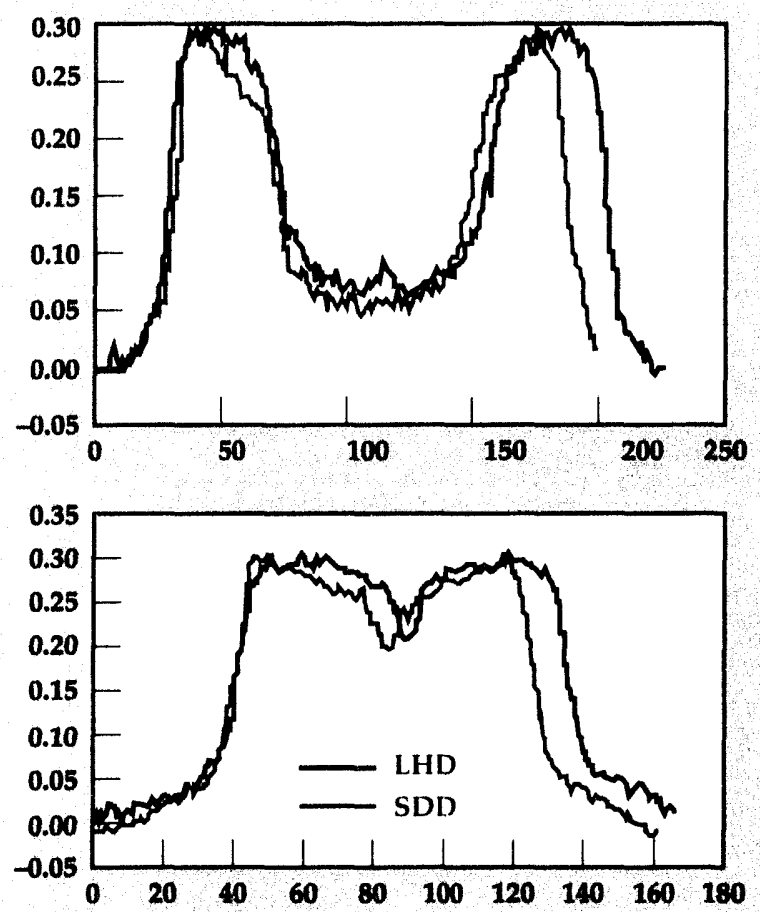

Figure 9. Comparison of enhancement in (a) LHD glass scintillator and (b) SDD fiber-optic scintillator for a plnch weld. 1-D profiles through the center of each image to the left are shown to the right.

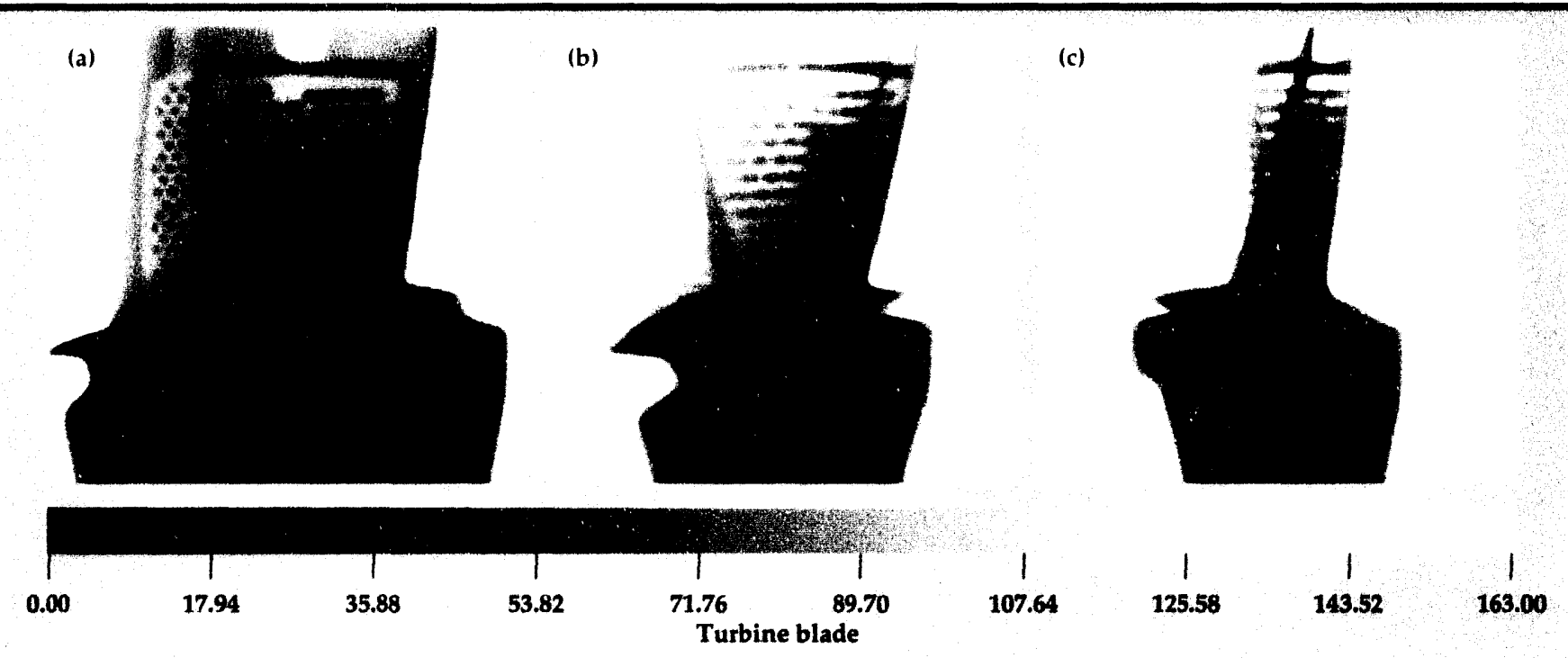

Fgure 10. Representative mediumenergy radiographs of turbine blade at (a) 0 , (b) $45^{\circ}$, and (c) 90 . 
The results of the performance of the LHD glass at higher energies is shown in a pair of digital radiographs of a 5.0-cm doublet, single-crystal turbine blade acquired at both 4 and $9 \mathrm{MeV}$, using two different Linatron sources and a lens-coupled, Cohu 4910, CCD camera-based detector system (Fig. 11). The pedestals and features of the blade are on the order of $1 \mathrm{~mm}$ in spatial extent. More detailed explanation of the turbine blade study is given below. We are attempting to further quantify the spatial resolution limits of this new glass for both medium- and high-energy CT scanning applications. Our preliminary CT results for highspatial-resolution, high-energy applications have been encouraging. Figure $\mathbf{1 2}$ is a sample of images

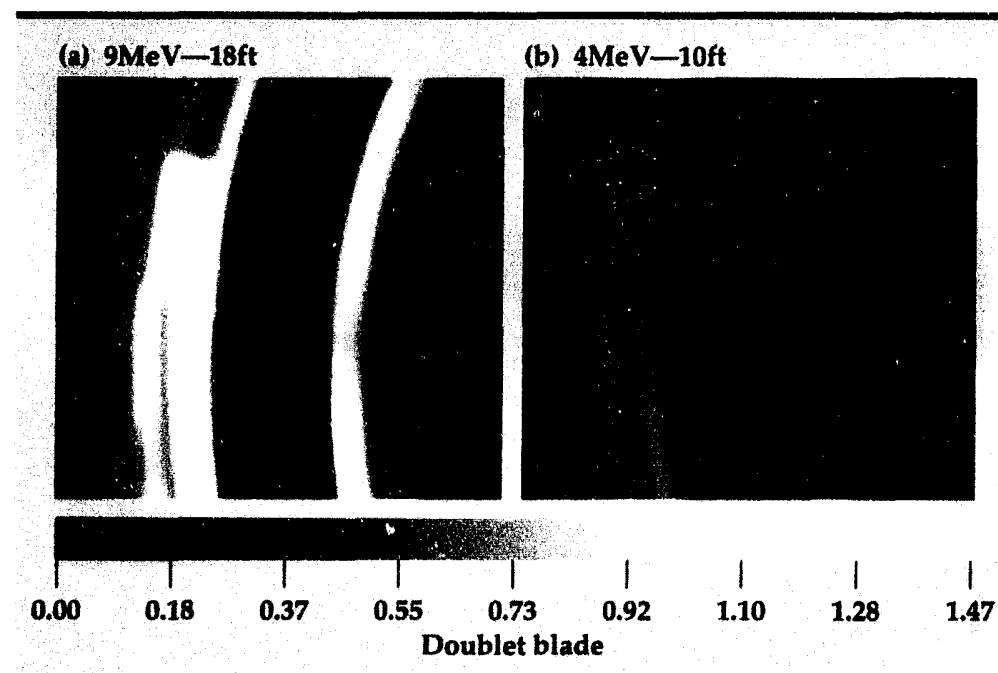

Flgure 11. Represontative highenergy radiographs of a doublet single-crystal turbine blade at (a) $4 \mathrm{MeV}$ and (b) $9 \mathrm{MeV}$.

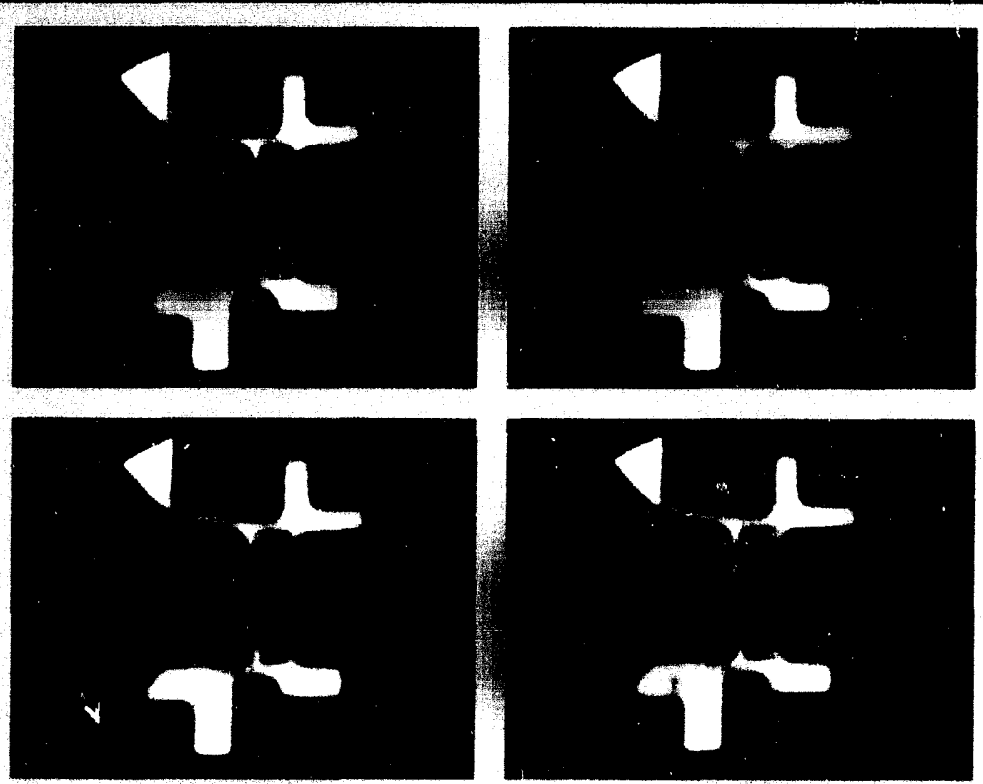

Figure 12. Representattve 2-D images from a volume (3D) CT data set of the doublet singlecrystal turbine blade, acquired using the HECAT detector and a 9MeV LNAC sounce. from a 3-D cube of data acquired with a 9-MeV Linatron on HECAT; they show the doublet turbine blade, measuring wall thicknesses of $500 \mu \mathrm{m}$.

In the next year, we will better quantify the spatial and contrast resolution performance of this new glass and the improvements this yields for lens-coupled, cone-beam CT scanners. With this proof-of-principle work as a base, and in cooperation with LLNL physicists working in astronomy, we are assembling a high-performance $\mathrm{CCD}$ camera with 14 to 16 bits and $2048 \times 2048$ detector elements, which can further explore the properties of this glass, and provide a higher performance, 2-D, cone-beam CT scanner. We will also examine this glass in slit-collimated configurations and with linear-array detectors as a means of obtaining highcontrast, high-spatial-resolution images that include less scatter.

Cone-Beam CT Reconstruction Technologies. We have implemented cone-beam reconstruction methods of others $21-25$ and developed our own, all with good results. 1,2 Last year, one men ber of our research team (S. Azevedo) worked in France at LETI on new cone-beam methods that benefited both $C T$ projects. New reconstruction algorithms and scanning methodologies were developed during the course of this collaboration, and a patent is pending in conjunction with the French government. 26 This and other cone-beam work are continuing in FY-93. Below, we describe some of the progress during FY-92 in image-reconstruction technologies, including region-of-interest conebeam CT, axi-symmetric CT, reverse cone-beam geometry, and a fast image-reconstruction processor.

Region-of-interest cone-beam CT. It is often necessary to view a part of an object at higher magnification than is needed over the rest of the object. Also, sometimes the object is too large for our conebeam scanner. In these cases, the data we acquire will be 'limited'; i.e., there will be missing ray paths from our projection measurements. This type of reconstruction problem is called 'region-of-interest' (ROI) CT and is a common problem in medical and industrial imaging. There have been solutions proposed for 2-D ROl imaging, but not for the 3-D cone-beam case.

Our algorithm for reconstructing cone-beam ROI data, called Radon-ROI, uses mathematical methods similar to the Grangeat method of conebeam image reconstruction. In Grangeat's method, the 2-D radiographic projections are mathematically converted (through weighting, filters, and re-binning steps) to an intermediate mathematical space known as the 3-D Radon domain. From this space, reconstruction of the volumetric 
image is straightforward, requiring only two sets of backprojections. The Radon space is an ideal place for combining data of different resolution, so it is ideal for ROl imaging. Two scans of the same object are acquired at different resolutions, a lowresolution scan covering the entire object and a high-resolution scan covering only the $\mathrm{ROI}$. These two scans are combined to form a single Radon space, which is reconstructed by the latter part of Grangeat's algorithm to form a final volume that displays the $\mathrm{ROI}$ at higher resolution than the surrounding part, without significant artifacts.

An example of the use of this method for $\mathrm{ROI}$ scanning of an automotive precombustion chamber is shown in Fig. 13. The image in Fig. 13a shows a single reconstructed slice through the object taken at low resolution. The data were acquired and reconstructed using cone-beam CT methods. The object has some shrinkage cracks just barely visible in the interior. A second scan of the precombustion chamber was acquired at a higher spatial resolution, but of the ROI only. The two scans were combined and reconstructed into a second image, as shown in Fig. 13b. The ROI area of this second image reveals the much higher spatial resolution obtained by this novel method.27

Axi-symmetric CT. We have applied the conebeam $C T$ reconstruction methods to the problem of obtaining 3-D exterior and interior information from axi-symmetric objects with only one 2-D radiograph or projection. This has application in several areas, such as manufacturing and high explosives testing. The problem is to perform conebeam $C T$ reconstruction of an object that has quasi-axial symmetry from a single radiographic view. For example, a high explosive can be radiographed during firing, with a flash $x$ ray unit, but only one view is available. If we assume axial symmetry, greater information can be gathered from the single radiographic view. We have performed conebeam image reconstructions of such tests and of simulated data to better understand the combustion mechanisms. Simulations provide useful information as to what kinds of artifacts to expect from any asymmetries in the object.

Another example is on-line monitoring of highvolume, axi-symmetric industrial parts. As an example, we applied this technique to a diesel engine piston. A single radiographic view of the piston was obtained (Fig. 14a) and reconstructed into a volume image. A representative 2-D cross section of this resultant volume is shown in Fig. 14b. With many such pistons being fabricated (as many as 2000 per hour), complete CT methods may be impossibly time-consuming. Howerer, using the

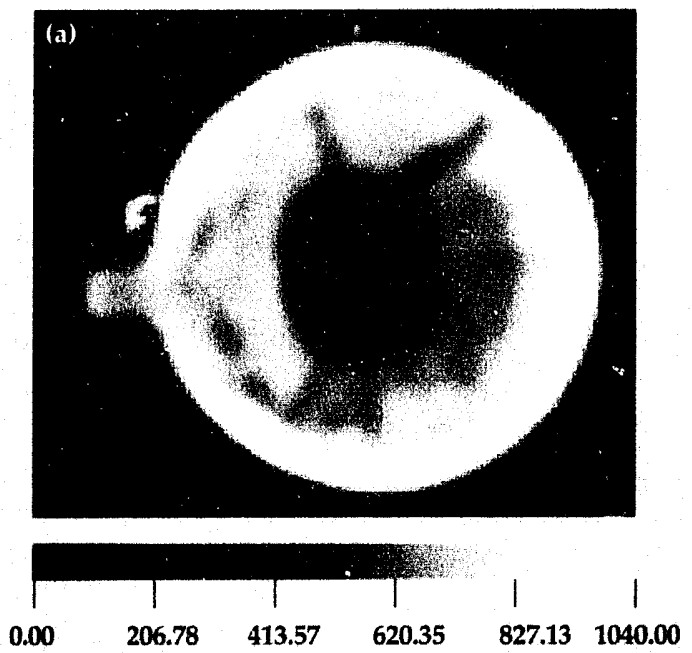

Figure 13. ROI image showing (a) single reconstructed slice at low resolution and (b) combined lowand high-resolution data reconstructed image. This is one slice out of a 3-D volume acquired by cone-beam methods. Notice the higher spatial resolution in (b). (Data courtesy of LETI, Grenoble, France.)
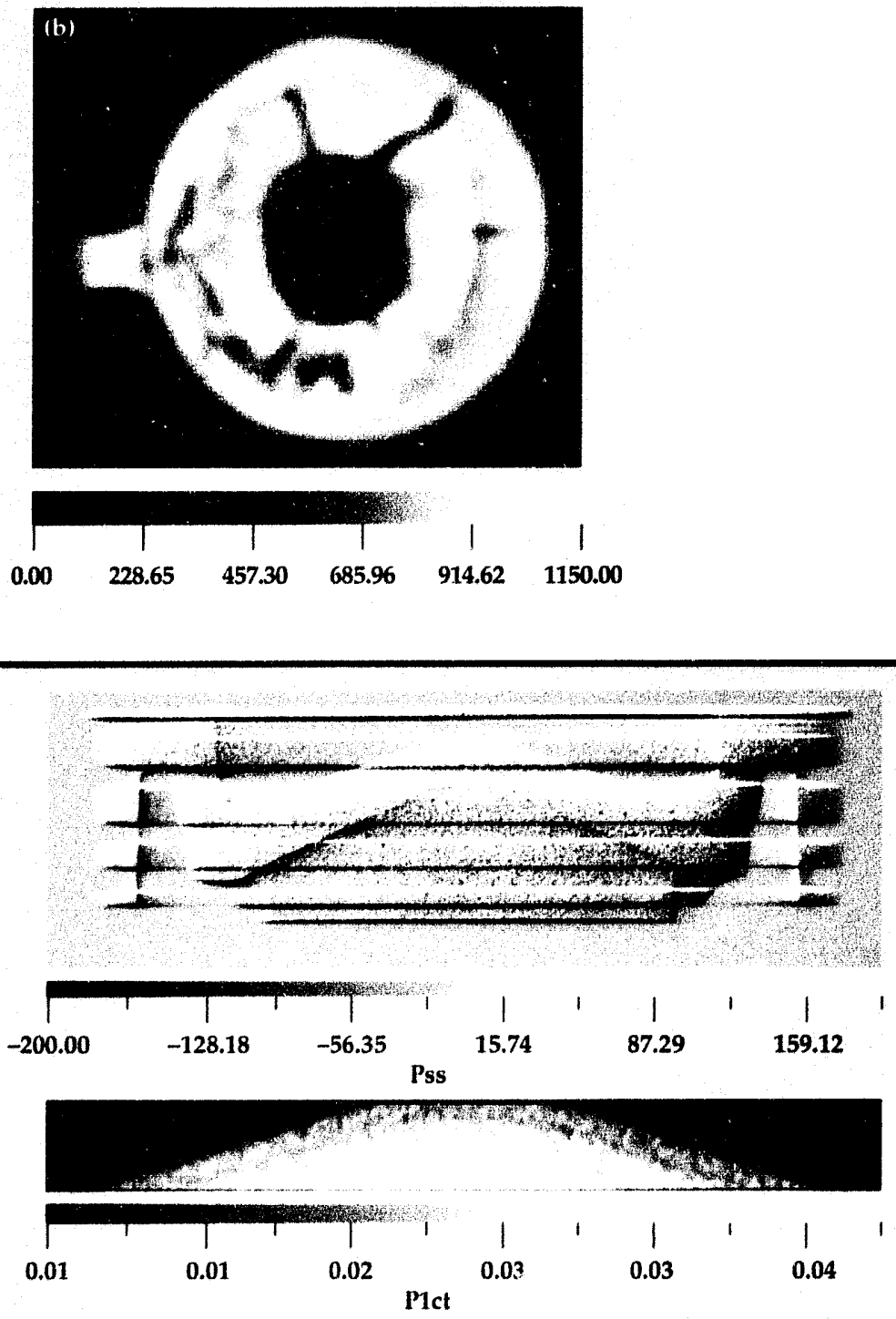

Figure 14. Representative (a) 2-D projection radiograph and (b) 2-D Cr images of a diesel engine piston. The data are extracted from a volumetric image obtained from a single 2-D view. 
above cone-beam reconstruction method, a single radiograph can display much more useful information about the part. For example, in Fig. 14b, small cracks within the interface between two different materials are much more visible than from the radiograph shown in Fig. 14a.

Reverse cone-beam geometry. A small company in San Ramon, California, called DigiRay, has developed a new radiographic method called "reverse geometry" cone-beam radiography. In this method, the source is a 2-D, raster-scanned flat panel, while the detector is a single element. The source raster defines the acquisition geometry, which is essentially a cone (Fig. 15). This system is unique and provides some inherent advantages over conventional, cone-beam, $x$ ray-imaging systems. The
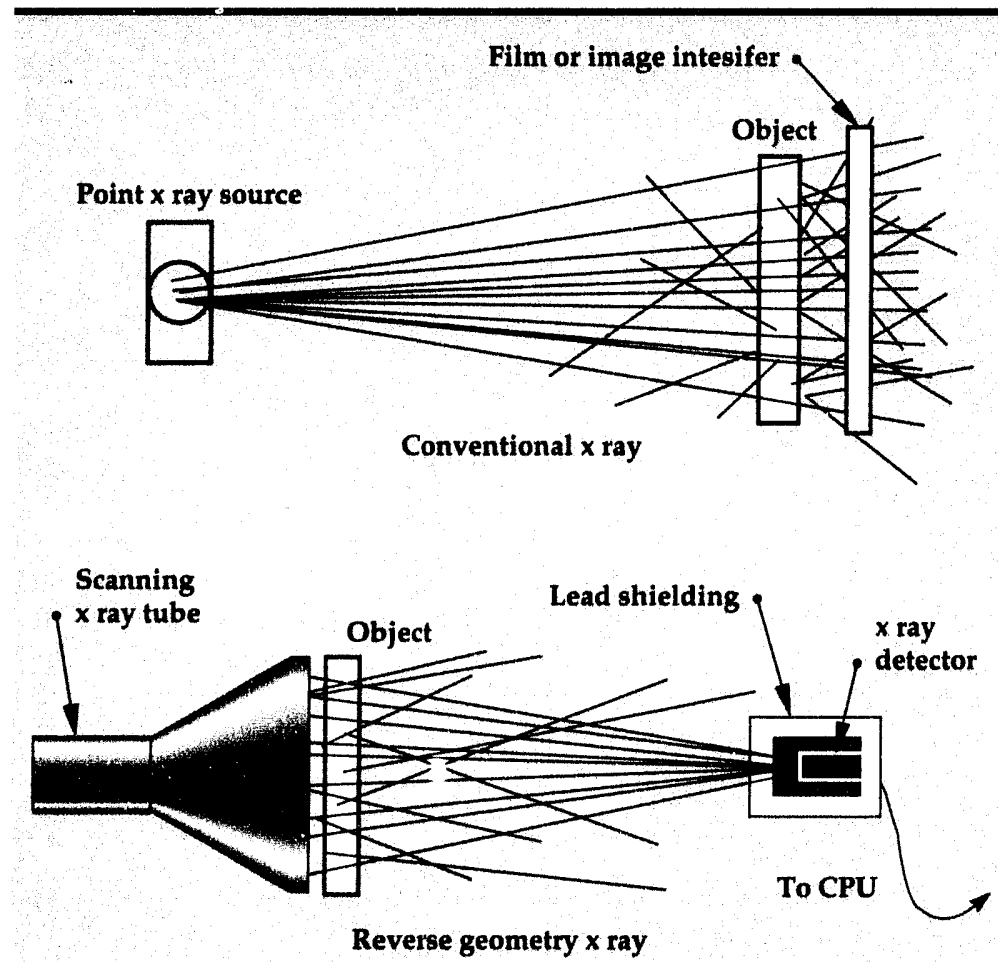

Figure 15. Comparison between (a) conventional cone-beam-projections dataracqutsition and (b) reverse geometry, cone-beam-projection data-acquisition systems.

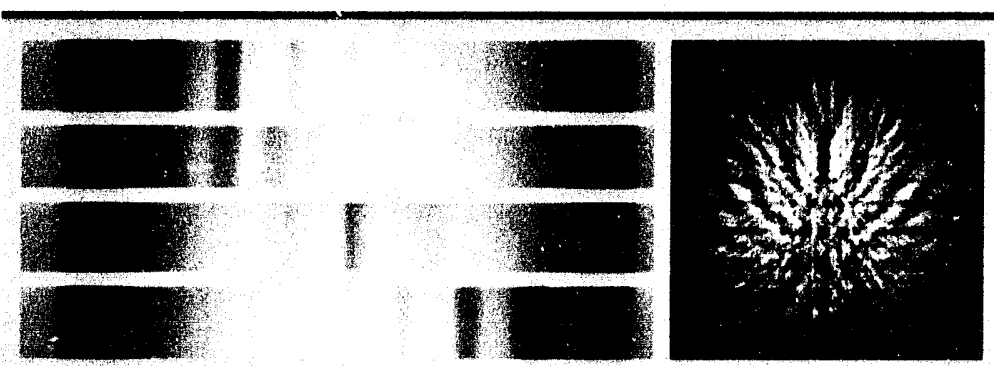

Fgure 16. Representative (a) projection data and (b) CT image of a MTF phantom using reverse geometry data acquisition. (Projestion data courtesy of Digiray, San Ramon, Califomia.) use of a converging beam has the potential to produce 2-D $x$ ray transmission images with little to no scattered photons. The DigiRay system also includes a $\mathrm{NaI}(\mathrm{Tl})$ detector that could be configured to acquire energy-specific data. Until recently, this system has been used exclusively for industrial radiographic applications, not for CT.

We have been evaluating the efficacy of using their unique method ${ }^{28}$ for industrial, cone-beam, CT imaging applications. DigiRay acquired 242-D, inverse-geometry, cone-beam projection images as a function of angle (every $15^{\circ}$ ) for a lexan modulation-transfer-function (MTF) phantom. Representative 2-D projections are shown in Fig. 16. These projection data were reconstructed using a parallel reconstruction algorithm. A resultant CT image is shown in Fig. 16. It is difficult to determine just how' useful the inverse geometry scans are from the $C T$ images, since these data have moire artifacts, due to the limited number of angular projections, that mask the scan results. In spite of these results, we still expect that this type of system could produce high quality 3-D imaging with little to no scattering artifacts. Weare working with DigiRay to set up an experiment in which we can obtain more angular projection data that should result in CT-reconstructed images without moiré artifacts. With the use of an energy-discriminating detector system, further enhancements are expected into the regime of materials characterization.

Reconstruction lurdtuare. Another important problem in cone-beam CT imaging is the speed of the image-reconstruction codes and architecture used. We have addressed this problem in a joint research project with a private company, Advanced Research and Applications Corporation (ARACOR) of Sunnyvale, California. In this project, ${ }^{24}$ an advanced computational engine, called the Konoscope reconstructor, was designed to reconstruct large cone-beam data sets in reasonable computation times (within $2 \mathrm{~h}$ of data-acquisition times) while being low in cost. This design was successfully completed in FY-91 and was realized as a hybrid, parallel, multi-processing system.

In the last year, we have purchased, assembled, and tested the basic building blocks of this system. This prototype was constructed to demonstrate and measure the performance of various algorithms operating on the reconstructor. Several conebeam reconstruction algorithms will be coded on the system for evaluation during FY-93. Also, the system will be used to evaluate other image-processing algorithms that may benefit from this unique design. 


\section{High-Energy CT Research}

High-Energy CT Scanners. We completed the first version of the HECAT scanner in FY-92. This scanner incorporated the features and flexibility of the video-camera-based CT (VIDCT), area detector software, and built upon our past experience with film radiography using 4- and 9-MeV VARIAN Linatron sources. HECAT is currently an area detector-based scanner that can acquire data in either a variable integration (typically from 2 to $10 \mathrm{~s}$ ), or RS-170 video-frame-rate, data-acquisition mode. Two different area detector systems have been used to acquire the CT projection data: (1) a VARIAN ER210, image-intensified SIT camera and (2) a COHU 4910 CCD camera. Both are lenscoupled to either a fiber-optic scintillator bundle or to a piece of the LHD clear glass via a visiblelight $90^{\circ}$ bending mirror. We have used the HECAT scanner to perform 3-D CT inspections of bridge members, engine parts, ceramic-metal castings, and single-crystal turbine blades.

CT systems contain four components: (1) source, (2) detector, (3) object manipulator, and (4) data acquisition and image-reconstruction and analysiscomputer. One of the challenges for a high-energy CT system is to reduce the effect of source blur (a blur from a finite source-spot size). High-energy sources typically involve relatively large source-spot sizes ( $2 \mathrm{~mm}$ in our case). Source blur, to first order, increases linearly with $x$ ray magnification.

A good rule of thumb is that the source blur, $\delta$, is equal to the spot size, A, times magnification, $M$, minus one, i.e., $\delta=A(M-1)$. Consequently, the source blur due $t_{0}$ finite spot size ${ }^{19}$ can be quite large at moderate magnification (nominally $1 \mathrm{~mm}$ at a magnification of 1.5). Large objects are difficult to position very close to the detector, by their very size. Also, object manipulators that can support 300 to $1000 \mathrm{lbs}$ are not small, and it is difficult to minimize the source detector distance if the manipulator cannot be fixed below the source detector. It is also useful to point out that to penetrate highly attenuating sections of an object, the source must be positioned close to the detector (to increase the effective flux per volume throughout the object), which increases the magnification for a fixed object to detector distance.

Recently developed objects (e.g., single-crystal turbine blades) are small, but contain highly attenuating materials. Consequently, there is a need for high-energy, high-spatial-resolution CT scanners. This need will continue to increase as metal manufacturing achieves new levels of complexity and precision.
HECAT (Fig. 17) acrommodates these demands in a number of ways. First we developed a highly flexible object manipulator (or stage) interface, fixturing for two different object manipulators (1) a small, $15-\mathrm{cm}$ o.d., rotation-translation stage that can hold up to $25-\mathrm{kg}$ objects, and (2) a rotation, translation, elevation and tilt stage that can support up to $\sim 350 \mathrm{~kg}$ and added fixturing that enables the position of the stage and scintillator face front to be in a variety of positions. The small stage is approximately $7.6 \mathrm{~cm}$ from the scintillator face front, while the large stage, with a $46-\mathrm{cm}$ o.d. rotational table, is a minimum of $25.4 \mathrm{~cm}$ from the scintillator. The fixturing for the scintillator is adjustable for a travel of $30.5 \mathrm{~cm}$. The camera is seated on a NEWPORT optical rail, and can be adjusted up to $20 \mathrm{~cm}$ inside the leaded enclosure to enable a variety of fields of view. Using these adjustments and different lenses, wecan obtain fields of view from $5 \times 5 \mathrm{~cm}$ to $28 \times 28 \mathrm{~cm}$. This scanner has been built with the flexibility to allow any object to be positioned as close to the detector as physically allowable. We have performed scans with this system, for different sized objects with cone angles up to $4.8^{\circ}$.

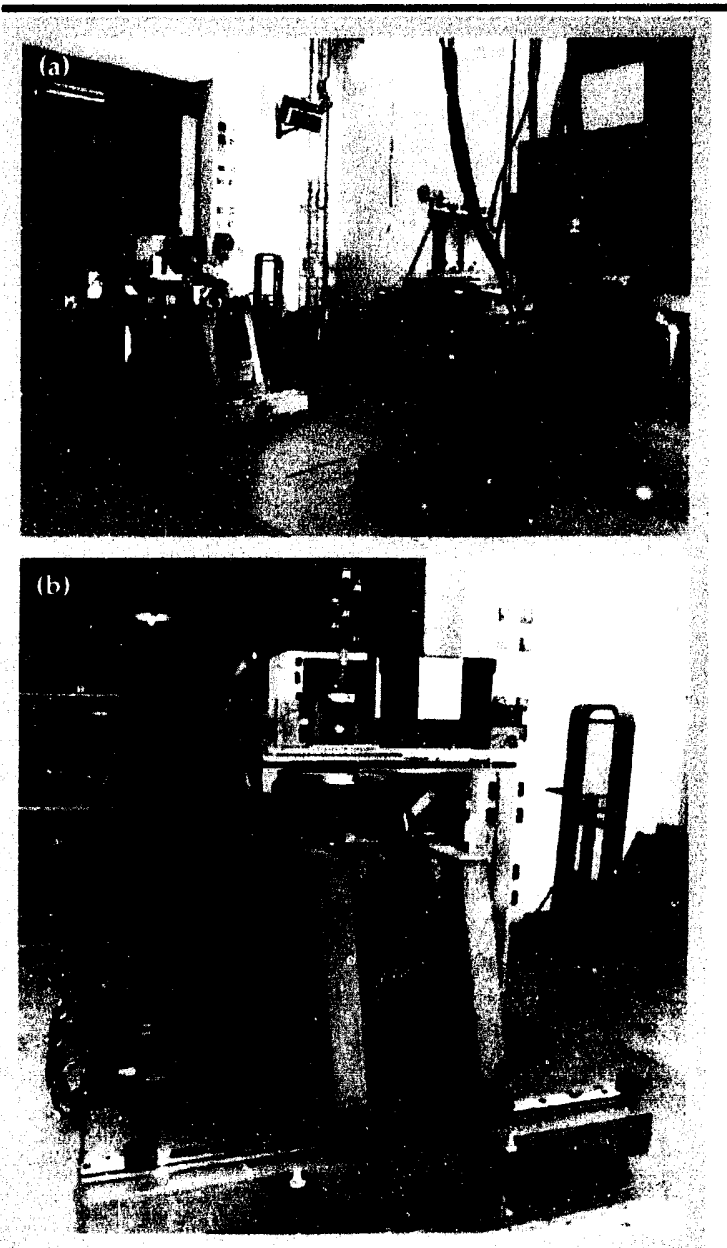

Figure 17. HECAT photos. 
This flexibility notwithstanding, one continuing limitation of this system is the relatively small maximum field of view $(28 \times 28 \mathrm{~cm})$ of the system as a whole. One of the advantages of the $9 \mathrm{McV}$ Linatron is the ability to penetrate objects with dimensions much greater than $28 \mathrm{~cm}$. The field-ofview limitation is only a result of third-generation

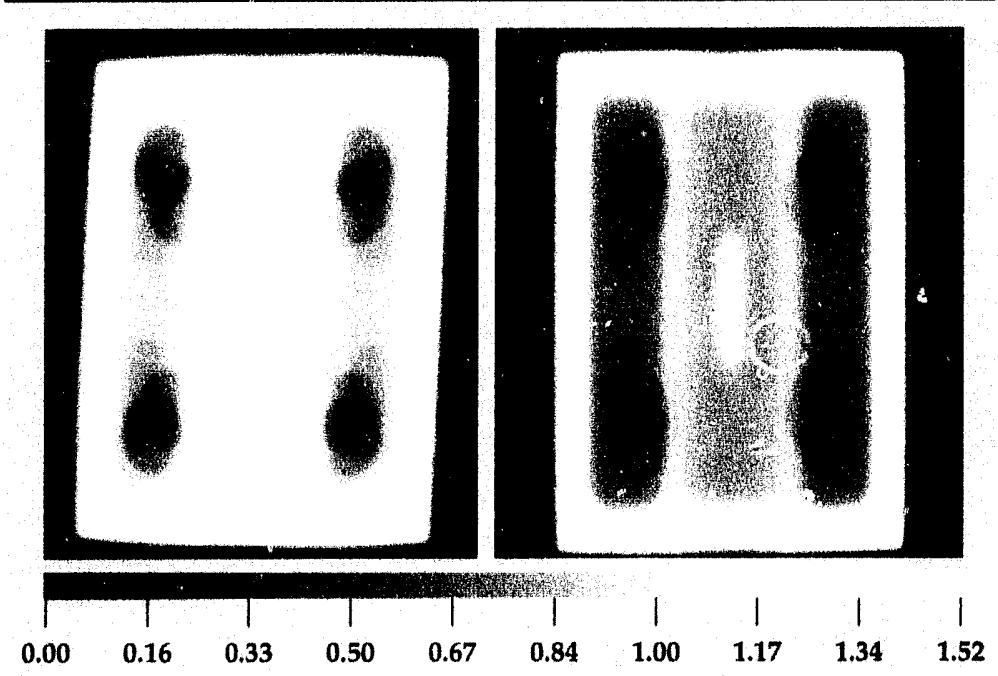

Figure 18. Representative digital radiographs of a prototype portliner from Caterpillar, Inc. The radiographs were obtained using a 9-MeV source with (a) the Varian ER210 camera-based detector; and (b) the Cohu camerabased detector. Each was lens-coupled to the LHD glass.

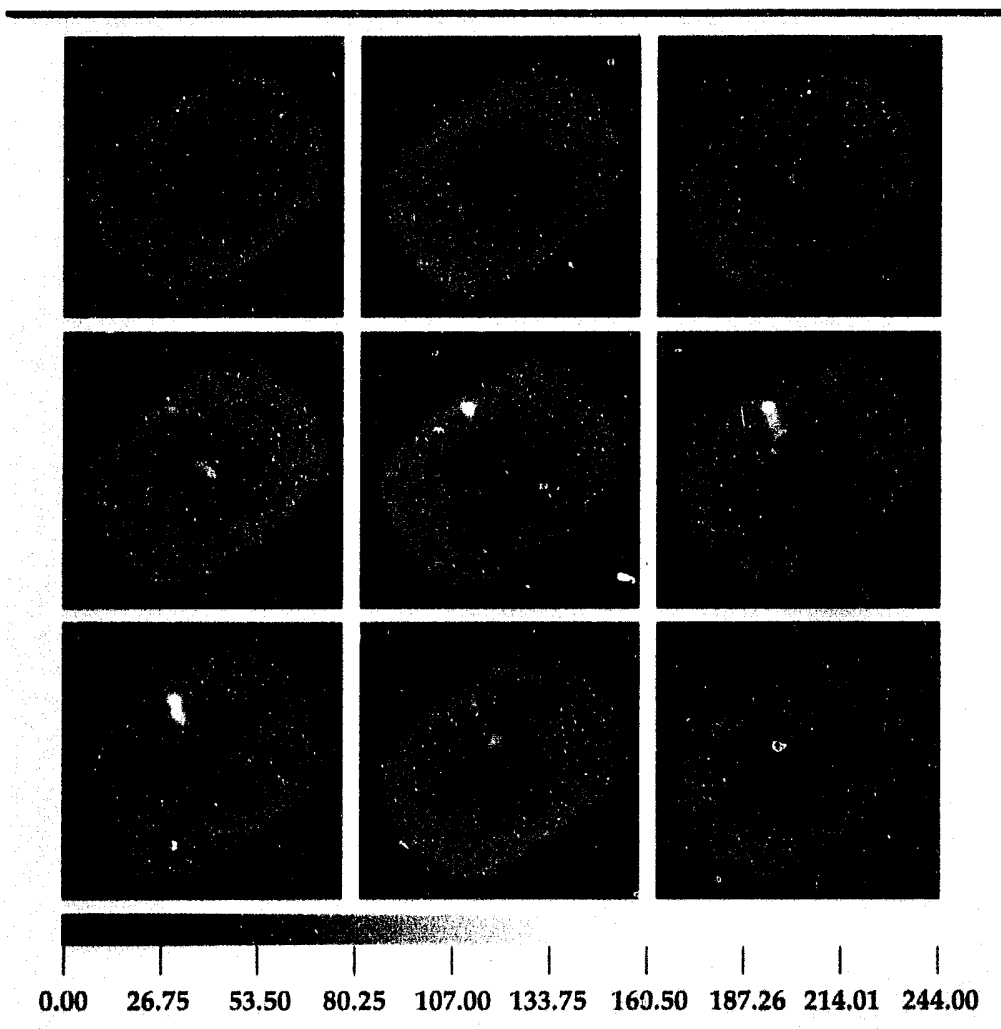

Figure 19. Representative 2-D images along the $z$ axis from a volume image of the track of a tungsten bullet. This data was acquired with the ER210 camera and the 4 MeV source. (rotation only) scanning geometry and can be overcome by implementing and applying second-generation (translation-rotation) scanning techniques. The latter technique extends the field of view to the total distance traveled of the object manipulator. We are pursuing this enhancement to extend the capability to HECAT in the next fiscal year.

We have used the HECAT scanner to directly evaluatedifferent high-energy scintillators and visible-light cameras. A comparison between the two HECAT cameras is summarized by two digital radiographs of a Caterpillar portliner. Both radiographs were acquired at $9 \mathrm{MeV}$ and are shown in Fig. 18. Note that the $C C D$ camera results have an increased performance over the SIT camera results.

High-Energy CT Applications. Most of our CT research has been within the low- $(6$ to $250 \mathrm{keV})$ to medium- $(250$ to $1,300 \mathrm{keV})$ energy range. We are now studying the effects of high-energy $x$ ray beams on the available detectors, and applications that require high energies for penetration.

One application for high-energy CT is the precision tracking of tungsten projectiles in target materials. Three-dimensional CT methods can generate dimensionally accurate images of the path of a projectile through a target in all three dimensions, showing the changes in trajectory and in the character of the projectile as it passes through the object. Figure 19 contains a set of 2-D images from a volume image in one orientation, while Fig. 20 is another set of 2-D images for a different orientation. From these images, it is particularly interesting to study just how the projectile changed its direction by $180^{\circ}$ to point in the direction of the initial momentum. This new capability provides an unambiguous image of the path of the projectile through the target medium.

Highenergy CT has been applied to various die wel engine components as a part of a Cooperative Research and Development Agreement between LLNL and Caterpillar, Inc. The goal of this project is to combine the NDE and computational resources and expertise available at LLNL, with the diesel-engine-design and manufacturing expertise of the Caterpillar Corporation to develop in-process monitoring and inspection techniques for diesel-engine combustion chamber components and materials. Early development of these techniques will assure the optimization of the manufacturing process by design/inspection interfaces. Project goals include (1) to improve the efficiency of diesel engines; (2) to meet or exceed new environmental regulations; and (3) to develop inspection and processis control technology for the production of advanced materials for improved diesel engines. 
Components tunderstudy range in size from 2-mm ().d. fuel injector tips to $30-\mathrm{cm}-x-36 \mathrm{~cm}-x-1.2 \mathrm{~m}$ cast iron exhaust manifolds. Masi of the effort to date has involved the interrogation of cast ironexhaust assemblies and protetypes (portliners). The largest of these devices is complex and non-symmetrical, with nominaloutsidedimensions of $30 \mathrm{~cm} \times .36 \mathrm{~cm} \times 1.2 \mathrm{~m}$. Our preliminary work has focused on a simple subsection of the exhaust manifold assembly, a 12-cm-x-15-cm-x-16-cm box wi han inner configuration of outlet holes and ceramic sleeving.

Our research has shown a substantial increase in spatial performance of the COHU C CD cameria as compared to the Varian ER210 with both coupled to the LHD glass (Fig. 18). The port rumning down the height of the object is lined with a ceramic material, which has numerous divets and cracks. A representative cross-sectional CT image of this object is shown in Fig. 21. The cross-sectional slice data reveal the ceramic-metal interface and features in the ceramic material.

\section{Additional CT Applications}

In this section, we describe some additional NDE research problems investigated during this fiscal year that are not published elsewhere.

Shape Charge. We have performed proot-ofprinciple CT scans on a conventional munitions shape charge to show how revealing $\mathrm{CT}$ is in identifying internal flaws nondestructively. To meet this end, we fabricated a few plastic inserts to mock air voids (four sets of hollow cylinders 1-,2-, 3-, and 4-mm diameter), and 1-and 2-mm mock separations of the explosive from the copper shape
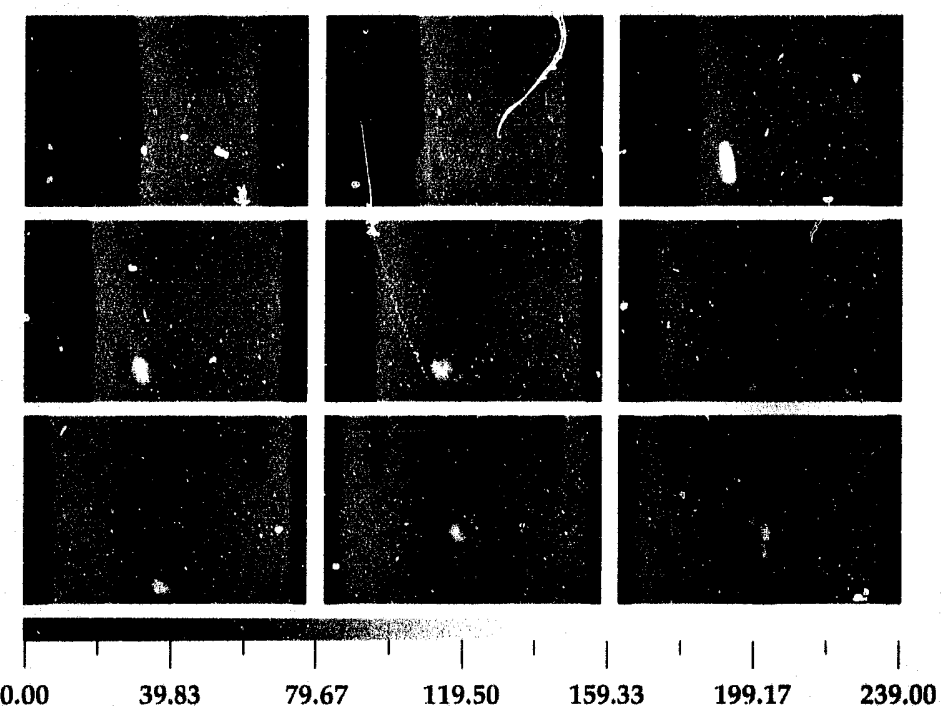

Figure 20. Representative 2-D images along the $y$ axis from the same volume image described in Fig. 19.

charge. These plastic inserts were fixed to the explosive side of the copper wall, and the charge was filled with a mock plastic explosive.

MECAT was used to acquire (T projection data of this shape charge at $70 \mathrm{~mm}$ from the bottom of the charge. A stummary of these results is shown in Fig. 22. The image on the left is the resultant image reconstructed from the projection data set. The CT image or tomogram represents a crosi-sectional view of the shape charge along its longritudinal axis, with $1-\mathrm{mm}$ spatial resolution and a slice-plane thickness of $1 \mathrm{~mm}$. The colorbar shown here in shades of gray relates colors in the image to the linear attenuation coefficient in $\mathrm{cm}^{-1}$.
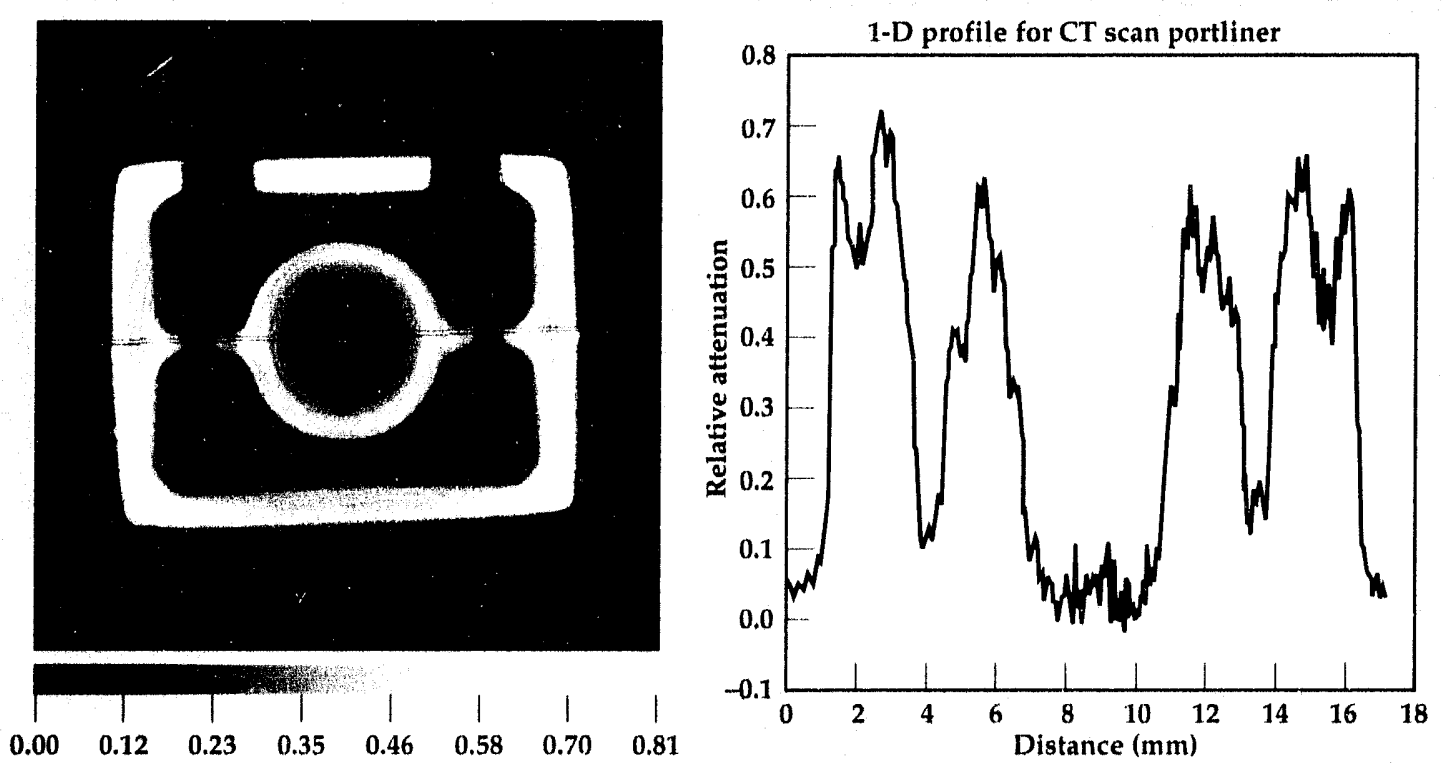

Figure 21. Representative 2-D image from a volume-image data set of the Caterpillar port liner. A 1-D profile shown by the black box is plotted to the right. 

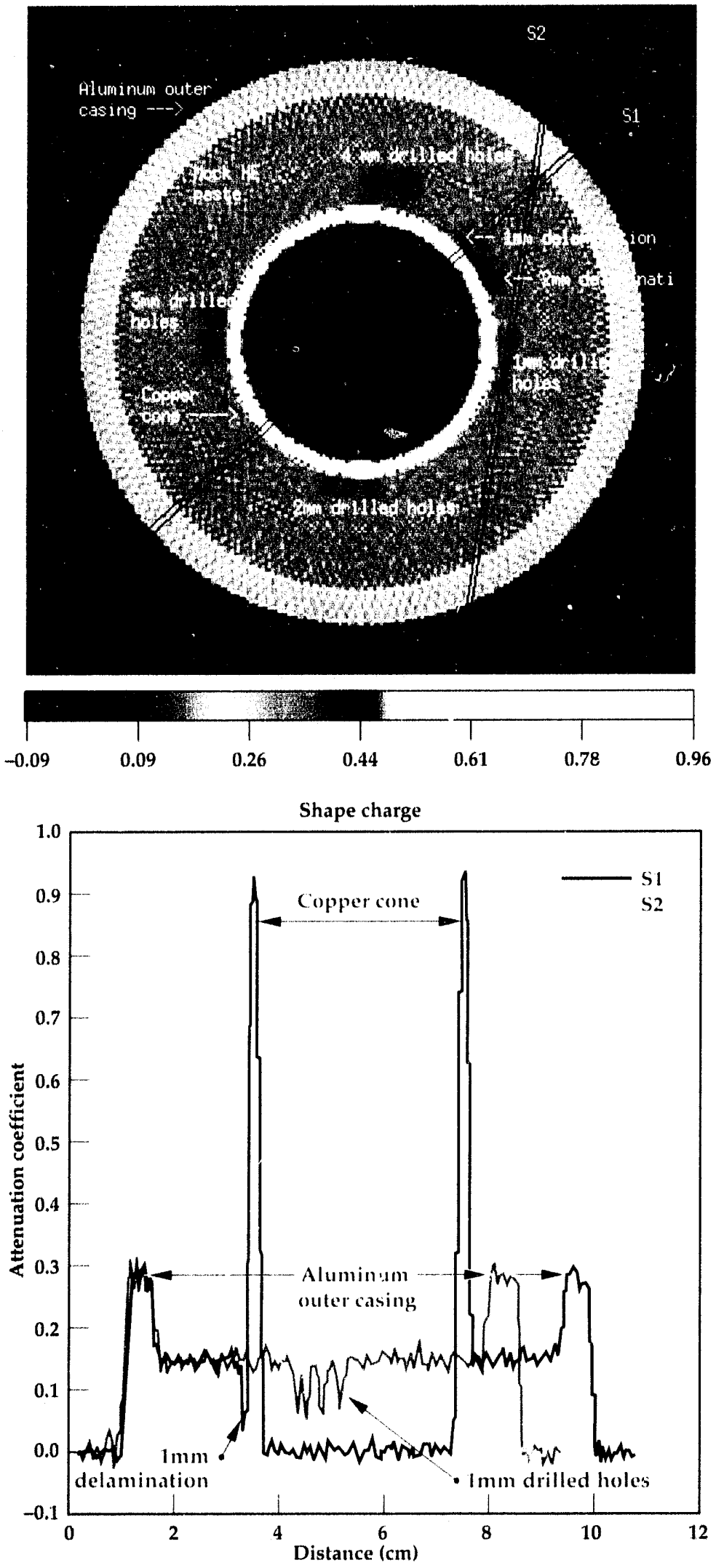

Figure 22. CT slice of a mock plastic-high-explosive shape charge with various defects. 1-D profiles are shown to the right.

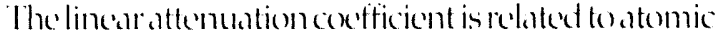
number and density of the stape charge. These results clearly reveal all four sets of voids and both the 1- and 2- $\mathrm{mm}$ separations between the copper wall and mock explosive. The tomographic image also provides the lecation and dimensions of both extermal and intermal details of the shape charge, which is sometimes very diffic ult, if not impossible, to obtain from radiographic data. With respect to Fig. 22, two 1-D profiles labeled in the image as S1 and S2 were extracted and are displayed in the plot to the right. The next step in this study would include the correlation of the CT images to actual performance of the shape charge, determined in live test firings:

Turbine Blades. Both high- and medium-eners. (T are being applied to a variety of singlecrystal, hollow-core turbine blactes (Fig. 23). These blates are required in motem high efficiency, low pollution jet engines where gas temperatures can exceed the melting point of the blade metal. Southwest Research Institute, Ciareett Engine, and the NDE Section at LLNL are combining resources and expertise to evaluate NDE techniques. The goals are to accurately dimension the internal geometries and detect anomalies intrinsic to hollow cast blades. Results of this project will be used to plan future funding actirities for development of adranced NDPE technicues. (T is a critical NDEE rechnicpue for turbine blate inspection due to their complex geometry and high aspect ratio, which limit the nature of internal information obtainatole from other technicues, such as radiography, infrared imaging, and ultrasonics. We are presently scanning each of the blades shown in Fig. 23, using the multitude of $\mathrm{CT}$ scommers discussed here

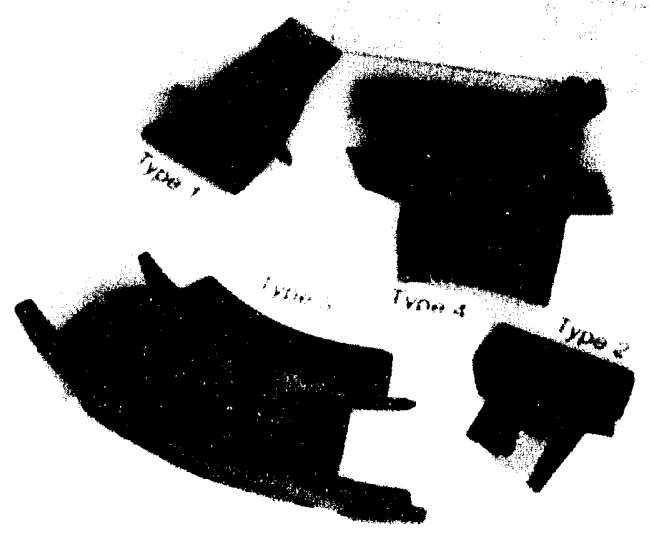

Figure 23. Photograph of four turbine blades provided by Wright Patterson Air Force Base for CT in.spection studies. 
and clsewhere. "Representative results are shown in Fig. 24 for the type 2 blacte using a mediumenergy source, and in Fig. 25 for the type 3 blade using a $9-\mathrm{MeV}$ Linac.

Bridge Cable CT Imaging. In cooperation with the California Department of Transportation and High Energy Services Corporation (HESCO), Woodside, California, proof-ef-principle experiments were performed in an attempt to image internal features of bridge cables and bridge cable terminuses. High-fidelity CT requires a rather large number of angular views (the ASTM recommends 1.5 times the size of the detector array in the horizontal direction). For a variety of reasons, most of which are related to functionality, components of civil brictges are highly attenuating with respect to $x$ ralys. Consequently, the internal inspection of these assemblies requires high energy (MV range) sources. Performing the radiography is complicated by the logistical issues regarding shielding the on-coming traffic from the radiation, or by developing reconstruction schemes that can obtain useful information from a limited number of views.

Using their in-depth knowledge of what can be done on bridges, HESCO acquired a limited-view CT projection (or radiographic) data set for application of our CT image-reconstruction techniques. The data set consisted of 24 film radiograph every 6 over 138. We developed interpolation schemes, in concurrence with other algorithm work, wo extend this data to 30 views over 180 ." (Other image-processing and image manipulation tools, developed for film (T, were then applied to obtain a reconstructed imagre of the cable. Figure 26 shows an example of the reconstructed images obtained using these methods. These results are encouriging enough to further pursue this method to inspect bridge cables.

Ancient Artifacts from Iraq. Wo have used CT to investigate two, corroded, ancient artifacts that were excavated in Iraq by University of Califomia Berkeley archaeologists under the direction of l'ofessor David Stronach. These artifacts, along with others, were exported to the U.S. with permission from the lraci archacological authorities for scientific analysis. The artifacts are believed to be objects used for persomal adornment. They were found on an ancient roadway of the Halzi Gate at the southeast corner of the city of Nineveh, the last capital of ancient Assy ria. The artifacts were found among skeletal remnants in the sack (destruction) level of Nineveh dating back to 612 BC, around the fall of the Assyrian Empire. It is believed that the skeletal remnants are from an immense battle fought there.

The encrusted artifacts were nondestructively evaluated using a quantitative CT scanner to learn about their original composition and geometry.
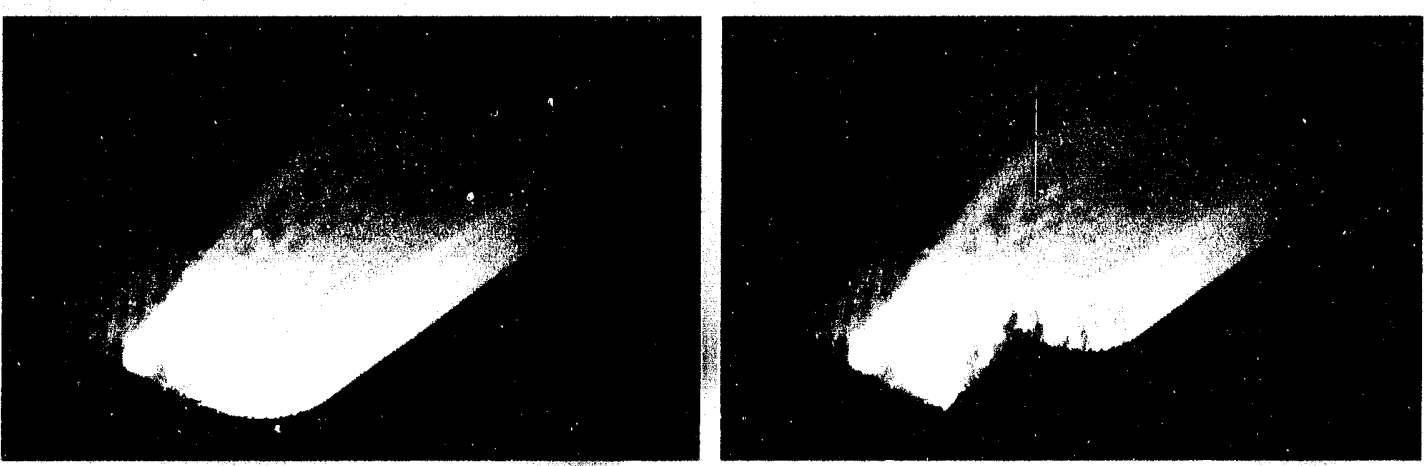

Figure 24. Representative CT images for the type 2 turbine blade, using a mediumenergy source at $270 \mathrm{kVp}$ and the Cohu camera cour pled to the LHD glass.
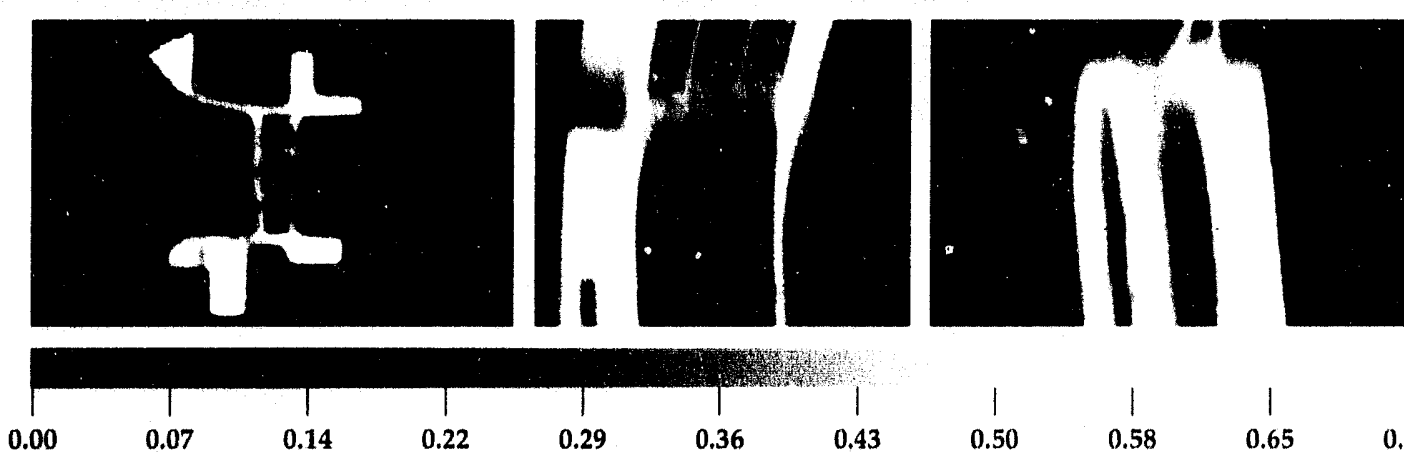

0.07

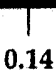

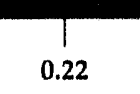

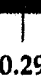

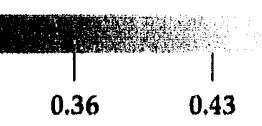

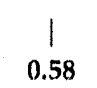

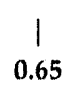

Figure 25. Representative $C T$ results for the type 3 turbine blade, using a 9-MeV Linac source and the Cohu camera coupled to the LHD glass. Shown are 2-D images along the $z$,

$x$, and $y$ axes, respectively, of a volume data set. 
Figure 26.

Representative radio graph and recon structed images of a bridge cable. The $\mathrm{CT}$ data consisted of 24 views over a range of 138 . (Radiographs courtest of HESCO. Woodside, California.)

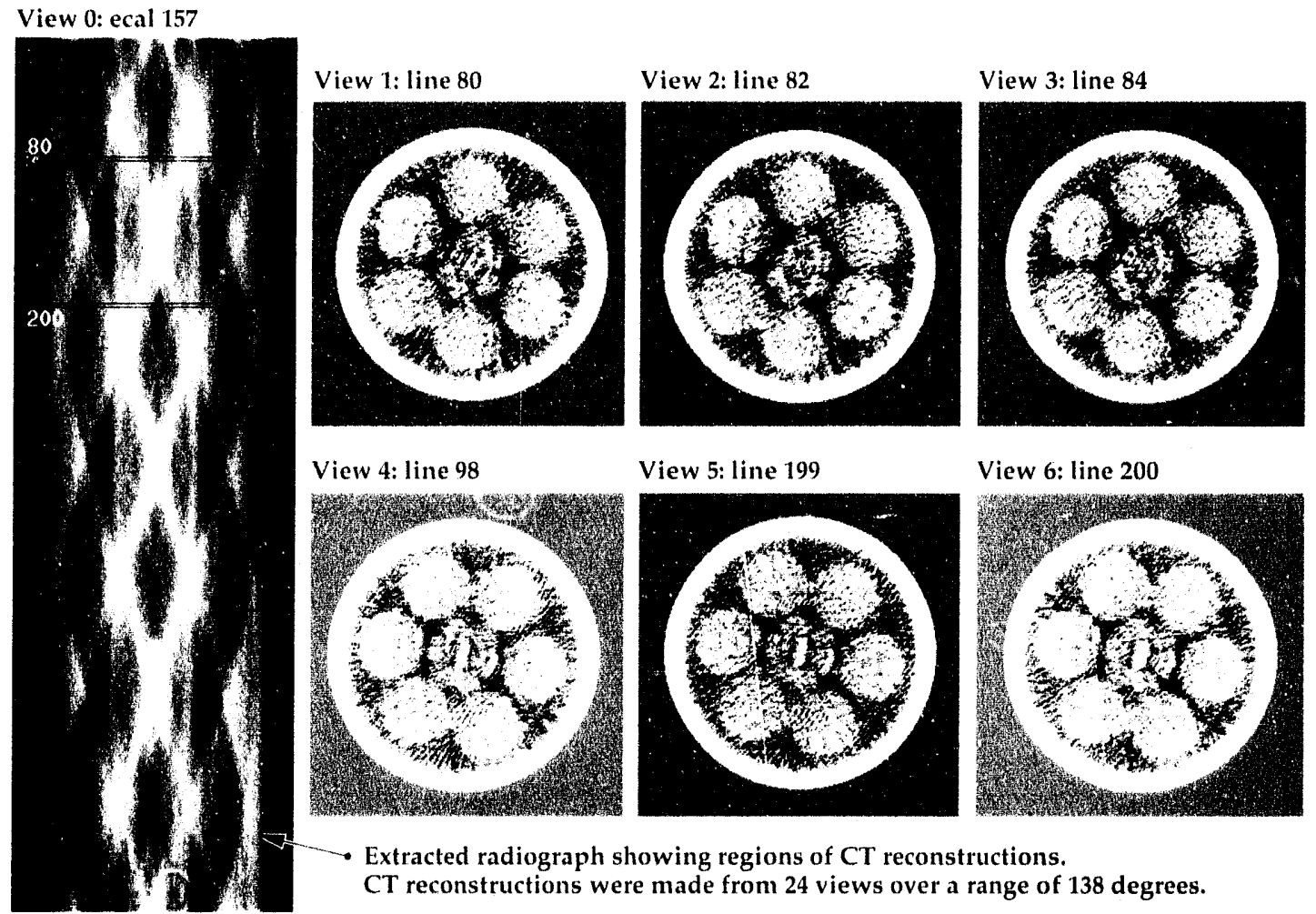

The two artilacts are shew in Fig. 27 and have the geometry of a cresecent moon and a ring. MEC $\mathrm{Al}$

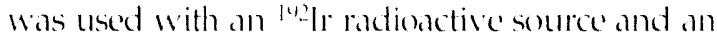
energy-discriminating, high-purity, germanium detector sistem. The "I data werecollected, with the ring placed within the concose edge of the crescent moom. A representative reconstructed image of a plane through the approximate midplane of the two artifats is shown in Fig. 28. The data used for reconstruction is the measurement of $x$ ray photem transmission from a narrow 317-keV peak of the 19-1r source. The reconstruction represents a spatially resolved image of photem absorption at this specific energy:

The CT images of these artifacts have helped the archaedegists determine the extent of comor sion sustained orer the vears. CT has provided information that can help determine what

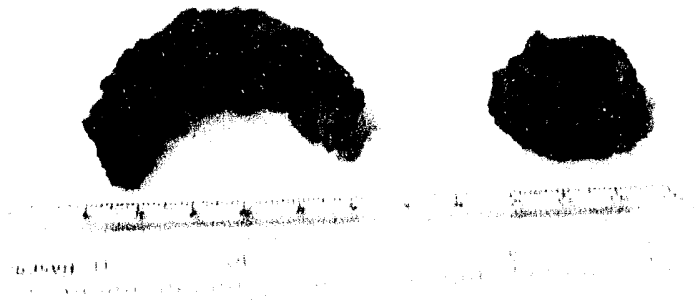

Figure 27. Ancient artifacts found in Nineveh, dated to 612 BC. material the artifacts are made of without destructively removing the encrusted substance around them. Also, the original geemetry of the artifacts can be extripolated from the CT images, without remowing the encrusted material.

() ver the last few years, we have used our various ( T systemson diverse experimental problems. Incadicase, ("Thasbeendemonstrated tobe anceffective forlformondestructive inspectionand/ (1) characterization. Wo will continue to improve C"I scanners built by our (T group and software tools extending the range of applications for CT technology: Weexpect tocontinue to participate in the tramsterofourtechnolegy within LLNL, around the department of fincergy nuclear weapons complex, and to commercial areas of investigation to assist in scaling up lo fiedtable industrial uses.

\section{Acknowledgements}

The authors would like to thank Christine Rob-

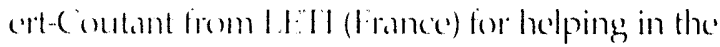

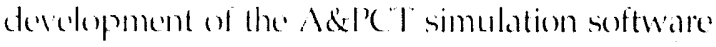
and in the andysis of the experimental A\&PCT data. We appreciate the many hepptul technical discuscioms with Bruce I lasegalla and Keenan

\section{Future Work}




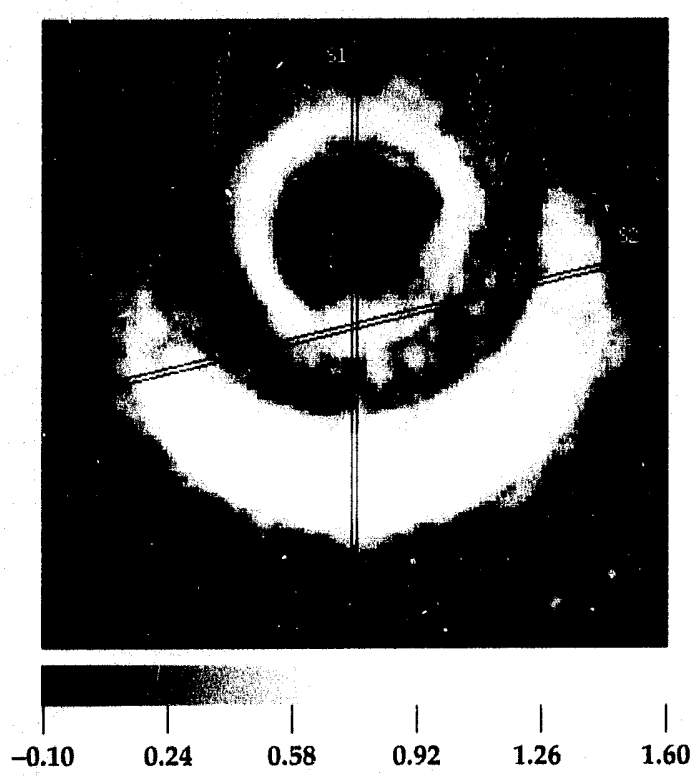

Brown at UCSF on A\&PCT simulation and experimental studies. Our discussions with Fred Cheu, General Motors, on the application of CT in the manufacturing environment have been very valuable in putting our research and development offorts in perspective. We thank Dick Albert at DigiRay for providing reverse-geometry conebeam data for us to reconstruct and analyze. We are grateful to Rich Whipple for filling the shape charge with a mock plastic explosive. Thanks to Mark Cutler and Willie Johnson at HESCO for providing the film radiographs of the bridge cable. We also would like to acknowledge the valuable contributions to this project by Linwood Hester, Earl Updike, Derrill Rikard, Jerry Haskins, Dave Camp, Zach Koenig, and Clint Logan. Many thanks go to the Engineering Research Committee for their continued funding and support of this project.

1. H.E. Martz, D.J. Schneberk, G.I? Roberson, and S.G. Azevedo, "Computed Tomography," Engimering Reserath. Derelopmint, and Tedmology, Lawrence Livermore National Laboratory, Livermore, California, UCRL-53868-9) , 7-6 (1492).

2. H.E. Martz, D.W. Perkins, S.G. Azevedo, D.J. Schneberk, M.F.Skeate, and C.P. Roberson, "Computerized Tomography," Engine'tring Resemole and Derelopment Thrust Area Report FY9o, Lawrence Livermore National Laboratory, Livermore, California, UCRL $-53868-90), 7-1+(1991)$.

3. S. Azevedo, Model-Based Computed Tommeraphy for Nomestruction Einluation, P’h.D. Thesis, University of California Davis, Davis, California (1991).

4. H.E. Martz, S.G. Azevedo, G.P. Roborson, D.J. Schneberk, and M.F. Skente, "Computerized Tomography," Finginering Resemble mint Deridop).

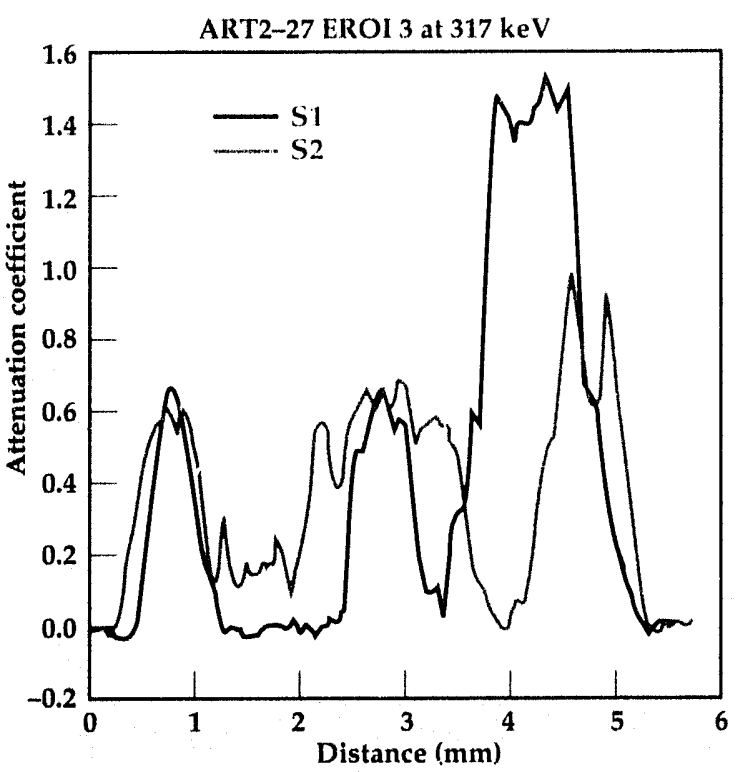

ment Thrust Aren Repert TY89, Lawrence Livermore National Laboratorv, Livermore, California, UCRL..-538(68-89, 7-15(149()).

5. H.E. Mart/, G.P. Roberson, and D.J. Sihneberk, "Progess in Computerized'lomography Sanners," Emergy and Tidmology Reriat, lawence Livermore Nationall aboratory, Livermore, California, UCRL 520001-90)-11.12 (November/December 1990).

6. H.E. Mart/, D.J. Schneberk, and C.I?. Roberson, "Applications of Computerized Tomography,"

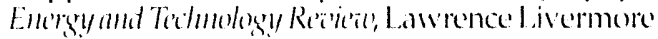
National Laboratory, Livermore, Califormia, UCRI $520001-9(1-11 \cdot 12$ (November/December 199()).

7. S.C. Azeredo, H.E. Mart/, J.M. Brase, and K.E. Waltien, "Computed Tomography," Enginesting

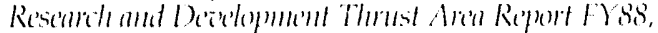
Lawrence Livermore National labonatory, I ivermore, California, UCRL-53868-88, 7-1 (1989).

8. I.M. Brase, K.I: Waltjen, and H.E.. Marts, "Com-

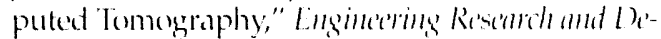

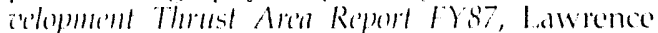
Livermore National L aboratory, I ivermore, California, UCRI - $538868-87,4-14$ (1988).

9. I.M. Brase, H.E. Marts, KE. Waltien, R.L. Hurd,

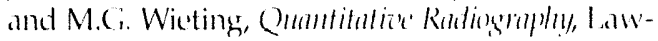
rence livermore National l aboratery, Livermore, California, UCID 19.323-86 (1986).

10. D.C. Camp, T.-F. Wang, and H.L: Mart/, "I'reliminary Minimum Detectable Limit Measturements in 208-1. Drums for Selected Actinide lsolopes in Mock-Waste Matrices," Pro. Tramsurmic Wask" Clantererization Conf., Idaho State University (Pocatello, ldaho), (August 1(1-12, |992); also) I awrena Livermone National I aboratorv, I ivermore, California, UCRI- $-\mid C-111345$ (14)2).

11. H.F. Marte, (i.l? Robersom, D).J. Shmeberk, and

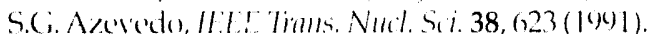

Figure 28. Representative reconstructed CT image of a plane through the approximate midplane of the ancient artifacts shown in Fig. 27. Two 1-D profiles are plotted to the right for further analysis of the L'T image data. 
12. G.P. Roberson, H.E. Martz, D.J. Schneberk, and C.L. Logan, "Nuclear-Spectroscopy Computerized Tomography Scanners," Proc. 1991 ASNT Spring Conf. (Oakland, California), 107 (March 18-22,1991).

13. H.E. Martz, G.P Roberson, C. Robert-Coutant, and D.C. Camp, "Experimental A\&PCT Research and Development Efforts To Characterize Mixed Waste Forms," Proc. Transuranic Waste Chameterization Conf., Idaho State University (Pocatello, Idaho), (August 10-12, 1992); also Lawrence Livermore National Laboratory, Livermore, California, UCRLJC-110826 (1992).

14. J.K. Brown, S.M. Reilly, B.H. Hasegawa, E.L. Gingold, T.F. Lang, and S.C. Liew, "Computer Simulation of an Emission-Transmission CT System," submitted to Met. Phys. (1992).

15. C. Robert-Coutant, H.E. Martz, and S.G. Azevedo, "Simulated A\&PCT Data To Study the Mixed Waste Forms Characterization Problem," Proc. Transuranic Waste Claracterization Conf., Idaho State University (Pocatello, Idaho), (August 10-12, 1992); also Lawrence Livermore National Laboratory, Livermore, California, UCRL-JC-110827 (1992).

16. R.C. Placious, D. Polansky, H. Berger, C. Bueno, C.L. Vosberg, R.A. Betz, and D.J. Rogerson, Mats. Eval., 1419 (November 1991).

17. R.C. Placious, D. Polansky, E.S. Gaynor, H. Berger, C. Bueno, R.A. Buchanan, C.L. Vosberg, and R.A. Betz, "An Improved Glass X Ray Scintillator," Final Report submitted to Naval Weapons Center, China Lake, California (1990).

18. A.H. Rodgers, Private communication, Synergistic Dector Designs, Mountain View, California (1992).

19. A.A. Harms and A. Zeilinger, Phlss. Met. Bio. 22(1), 70 (1977).
20. M. Barker, Private communication, I ackhered Missile and Space, Palo Alto, Califomia (1992).

21. L.A. Feldkamp, L.C. Davis, and J.W. Kress, JOSA $\Lambda$, 612 (1984).

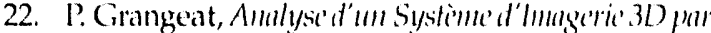

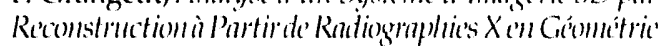
Comizue, Ph.D. Thesis, I'Ecole Nationale Superieure des Telecommunications, Grenoble, France (1987).

23. B.D. Smith, IEEE. Trans. Mot. Inuging, MI-4 (1), 14 (1985).

24. H. Kudo and T. Saito, $/ O S A \triangle 7,2169(1990)$.

25. Ph. Rizo, P. Grangeat, P. Sire, I'. LeMasson, and P. Melennec, IOSA A 8 (10), 1639 (1991).

26. S. Azevedo, P. Grangeat, and Ph. Rizo, "Procede de Reconstruction d'Images Tridimensionelles d'une Region d'Interet d'un Objet, Comprenant la Combinaison de Mesures surl'Ensemble de l'Objet a des Mesures sur une Region d'Interet de l'Objet, et Installation Appropriée," French Patent Application 92-11148, September 1992.

27. S. Azevedo, Ph. Rizo, and I'. Cirangeat, "Regionof-Interest Cone-beam Computed Tomography," submitted to JOSA A (1992).

28. R. Albert, Private communication, Digimy, San Ramon, California (1992)

29. S.G. Azevedo, H.E. Martz, and G.I. Roberson, "Computerized Tomography Reconstruction' Technologies," Encrgy and Tedmology Reriew, Lawrence Livermore National Laboratory, Livermore, California, UCRL-520)()-9)-11.12 (November/December 1990).

30. D.J. Schneberk it al., Limited Angle RadiographlyBased Computed Tomography for In-Situ lnspretions of Bridge Cables, to be published (1993). 


\title{
Laser Ceneration and Detection of Ultrasonic Energy
}

\author{
Graham H. Thomas \\ Engineering Sciences \\ Mechanical Engincering
}

We have developed a facility to generate and detect ultrasonic energy with lasers. Lasergenerated ultrasonics is an attractive alternative to traditional ultrasonic nondestructive evaluation (NDE), because it allows remote, noncontacting, ultrasonic NDE. We are developing NDE applications for use on contamination-sensitive components and in hostile environments. Laser ultrasonics has several other advantages, such as broadband excitation, multimode acoustic energy generation, and adaptability to scanning complex shapes.

\section{Introduction}

Ultrasonic nondestructive evaluation (NDE) is a valuable technology for material characterization and defect classification.' Laser-based ultrasonics allows us to explore many new applications. For example, laser ultrasonics can be performed in hostile environments, such as in a furnace or a glove box. We are also pursuing laser ultrasonic techniques for providing feedback control for processes such as welding, composite curing, and solid-state bonding. ${ }^{2}$

\section{Progress}

We have acquired ultrasonic data on a variety of specimens to test the system's capabilities. We have demonstrated the feasibility of laser ultrasonics to perform feedback control for directing a welding operation. We performed an experiment to show the ability of laser ultrasonics to measure the distance to a weld seam. The application we are considering is for laser welding, where the welding laser must precisely track the joint. Our approach is to rigidly fix the laser acoustic system to the welding beam. The laser acoustic system can accurately measure the distance between the location of acoustic generation and the weld seam. If the welding laser wanders off the seam, the ultrasonic path length will change (see Fig. 1). The path length change will be fed to the welding laser alignment controller to adjust the laser location.

We demonstrated the ability of laser ultrasonics to measure the distance between the ultrasonic

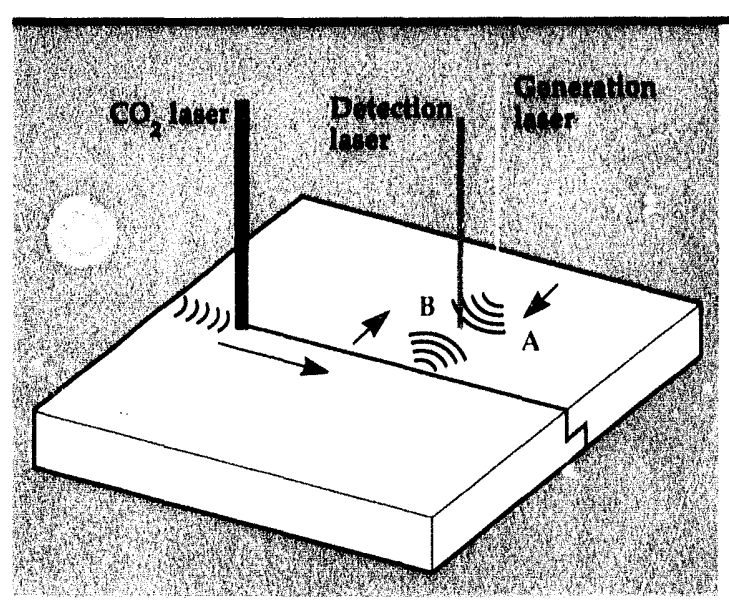

Figure 1. Diagram of laser ultrasonic feed back systom for controlling wolding $\mathrm{CO}_{2}$ laser allenmont. The ultrasonic surface wave is first sensed by the detection laser as it passes under the beam. The surface wave then reflects off soam ahoad of the wolder and is sensed agaln by the dotection laser on the rotum.

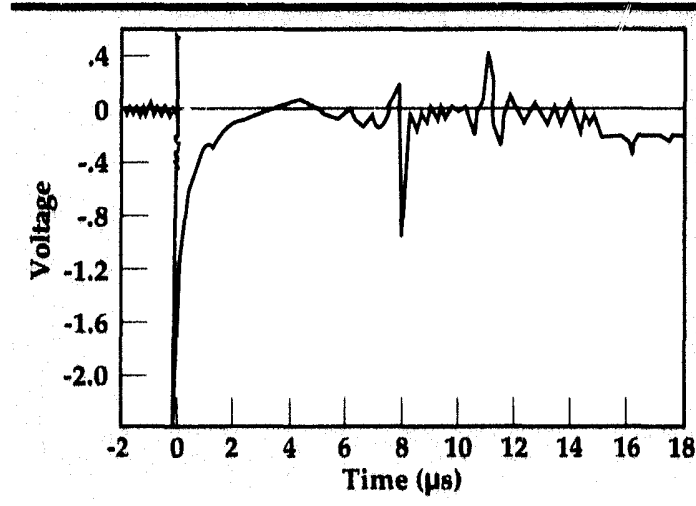

Figure 2. Sample results of laser ultra sonic signal from weld seam. TIme between pulses provides foedback to control welding laser tracking. surface-wave-generation location and the weld seam. We generated ultrasonic surface waves in surrogate specimens. Figure 2 displays an example of the ultrasonic signals, where the time between the two pulses is a function of the distance to the seam. If these pulses move relative to each 
other, the welding laser has moved off the seam. The timing between pulses should allow us to calculate position accuracy to $.001 \mathrm{in}$.

\section{Future Work}

We are increasing our knowledge of laser generation and detection of acoustic energy, and improving our laser acoustic facility. We are investigating applications of laser acoustics to NDE problems simultaneously at Lawrence Livermore National Laboratory and within U.S. industry. Specifically, we will continue to explore applications for laser acoustics to control selected manufactur- ing processes, such as welder alignment, composite curing, and plutonium processing. Since each application of laser-generated ultrasonics entails a customized system, we need to have a thorough understanding of the fundamental capabilities and limitations of the technology to design the optimal inspection facility.

1. J. Krautkramer and H. Krautkramer, Ultrasonic Testing of Materials, Springer-Verlag New York, Inc. (New York, New York), 1977.

2. N.M. Carlson and J.A. Johnson, "Laser Generation in a Weld Pool," Rerviene of Progress in Qunntitative" Nondestructive Evaluation 7B, Plenum Press (New York, New York), 1485 (1988). 


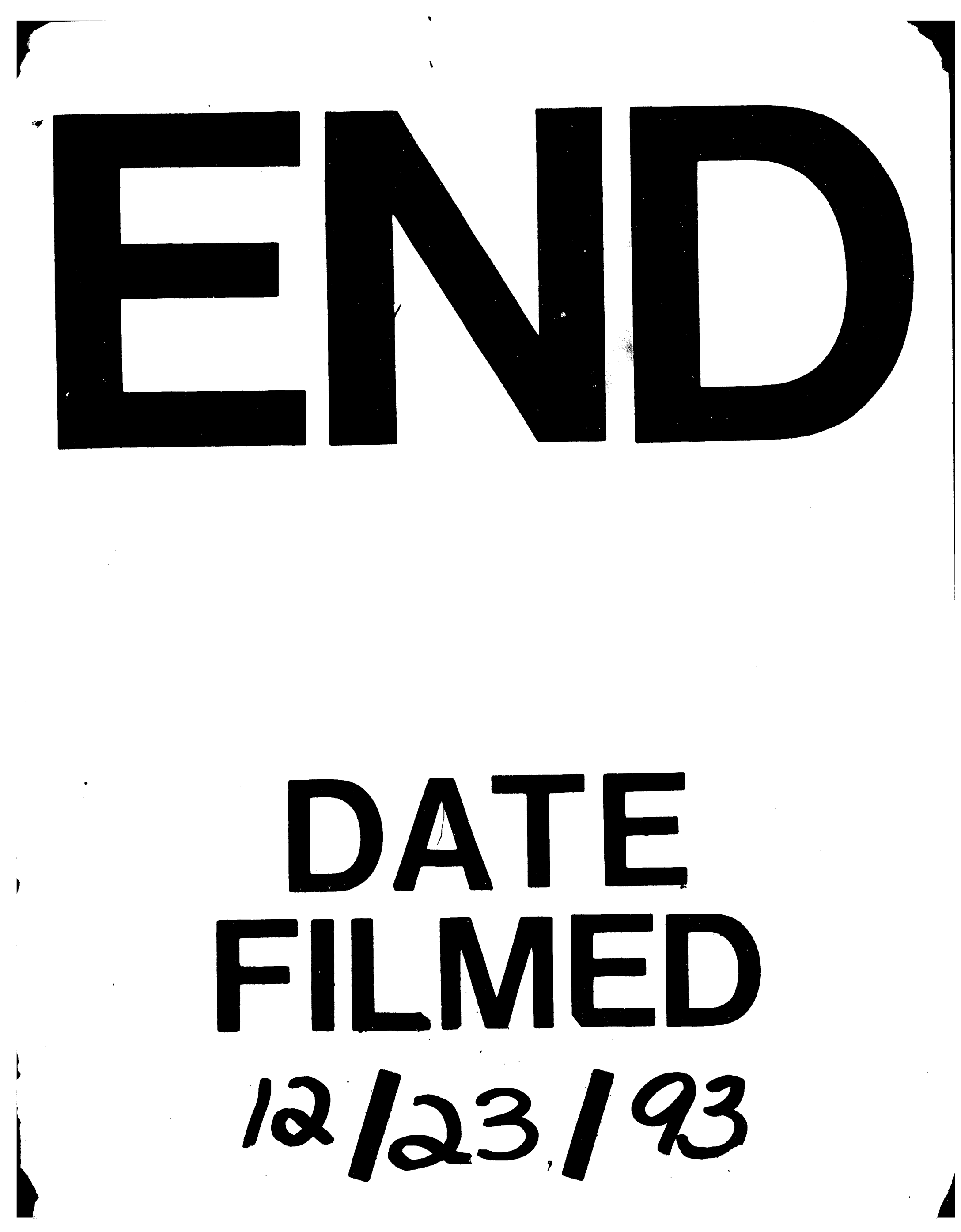




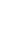

